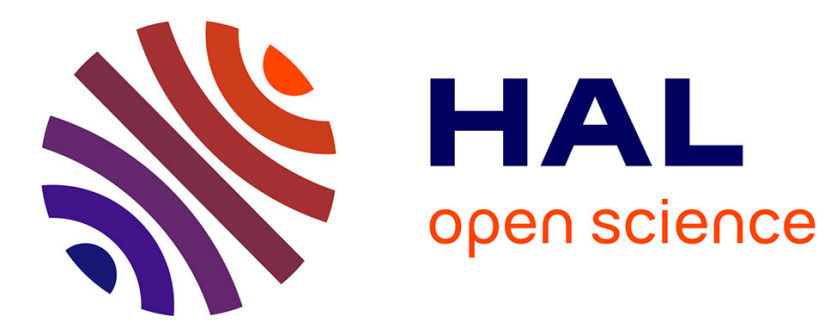

\title{
Spin-VCSELs with local optical anisotropies: toward terahertz polarization modulation
}

M. Drong, T. Fördös, H.Y. y Jaffrès, P. Ciompa, J. Peřina, K. Postava, J. Pištora, H.-J. Drouhin

\section{- To cite this version:}

M. Drong, T. Fördös, H.Y. y Jaffrès, P. Ciompa, J. Peřina, et al.. Spin-VCSELs with local optical anisotropies: toward terahertz polarization modulation. Physical Review Applied, 2020, 15 (1), 10.1103/PhysRevApplied.15.014041 . hal-03320871

\section{HAL Id: hal-03320871 https://hal.science/hal-03320871}

Submitted on 16 Aug 2021

HAL is a multi-disciplinary open access archive for the deposit and dissemination of scientific research documents, whether they are published or not. The documents may come from teaching and research institutions in France or abroad, or from public or private research centers.
L'archive ouverte pluridisciplinaire HAL, est destinée au dépôt et à la diffusion de documents scientifiques de niveau recherche, publiés ou non, émanant des établissements d'enseignement et de recherche français ou étrangers, des laboratoires publics ou privés. 


\title{
Polarization dynamics of Spin-VCSELs with local optical anisotropies
}

\author{
M. Drong, ${ }^{1,2,3, *}$ T. Fördös, ${ }^{2,3}$ H. Y. Jaffrès, ${ }^{4}$ P. Ciompa,,${ }^{2,3,4}$ \\ J. Peřina Jr., ${ }^{5}$ K. Postava, ${ }^{2,3}$ J. Pištora, ${ }^{2,3}$ and H.-J. Drouhin ${ }^{1}$ \\ ${ }^{1}$ Laboratoire des Solides Irradiés, École Polytechnique, CNRS, CEA-DSM-IRAMIS, \\ Institut Polytechnique de Paris, 91128 Palaiseau Cedex, France \\ ${ }^{2}$ Nanotechnology Centre, VSB-Technical University of Ostrava, \\ 17. listopadu 2172/15, 70800 Ostrava-Poruba, Czech Republic \\ ${ }^{3}$ IT4Innovations, VSB-Technical University of Ostrava, \\ 17. listopadu 2172/15, 70800 Ostrava-Poruba, Czech Republic \\ ${ }^{4}$ Unité Mixte de Physique CNRS/Thales and Université Paris-Saclay, \\ 1 Avenue A. Fresnel, 91767 Palaiseau Cedex, France \\ ${ }^{5}$ Joint Laboratory of Optics of Palacky University and Institute of Physics of Academy of Sciences of the Czech Republic, \\ Palacky University, 17. listopadu 12, 7720 07 Olomouc, Czech Republic
}

(Dated: October 19, 2020)

\begin{abstract}
We present a semi-classical model for spin-injected vertical-cavity surface-emitting lasers (spinVCSELs) with local optical anisotropies. Particular focus is put on highly-anisotropic spin-lasers with broad application potential. A generalized matrix formalism for extraction of the laser modes is introduced, which enables to calculate spatial distribution of vectorial modes in arbitrary spin-VCSELs. Time-dependence of such laser modes is further studied using the generalized coupled mode theory (CMT). It is the natural anisotropic generalization of the conventional modedecomposition approach. We use the circularly-polarized optical modes as the basis for CMT, which leads to extension of the well-known spin-flip model (SFM). In contrary to conventional SFM, the only input parameters are the geometric and local optical properties of the multilayer structure and properties of the gain media. The advantages of the theory are demonstrated on design and optimization of spin-VCSEL structure with high-contrast grating. We show that the proposed structures can be used for i) polarization modulation in $\mathrm{THz}$ range with tremendous applications for future ultrafast optical communication and ii) as perspective compact $\mathrm{THz}$ sources.
\end{abstract}

\section{INTRODUCTION}

The fundamental connection of electron and photon spin [1] has led to the development of the spin-polarized semiconductor lasers with VCSEL geometry $[2,3]$. In principle, the properties of such devices significantly depend on the degree of spin polarization in their gain media, which involves optical, electrical or hybrid spin injection $[4,5]$. Important advances have been achieved in this research field in the recent years. Namely, it has been shown, that VCSEL with spin-polarized gain media exhibit lower lasing threshold $[6,7]$ and allows direct polarization control $[8,9]$. Recently, the spin-amplification using spin-VCSEL has been demonstrated [10]. However, their most important premise these days seems to be the possibly ultrafast modulation dynamics, which has the potential to boost the capacity of optical communication systems [11]. This is possible due to the combination of two factors: an extremely fast spin-mixing rate in the semiconductor quantum wells (QWs) and strong linear optical anisotropies within the VCSEL cavity. It was demonstrated, that InAlGaAs QW VCSEL with modulated spin-injection is appropriate for high-speed data communication at telecom wavelength $(\lambda=1.55 \mu \mathrm{m})$ [12]. More recently, the experimental studies using $\lambda=$ $850 \mathrm{~nm}$ GaAs QW VCSEL showed, that spin modulation

* mariusz.drong@vsb.cz can significantly overcome the limitations of conventional VCSELs concerning modulation speed, even by one order of magnitude [13]. This is closely related to the possibility of generating the coherent $\mathrm{THz}$ radiation using highly-birefringent spin-VCSELs, based on interference of orthogonal linear modes of different frequencies. Such technological improvement would lead to development of the compact tunable $\mathrm{THz}$ sources operating at room temperatures [14].

Concerning steady-state properties, the first qualitative model taking into account both spin degree of freedom and cavity birefringence was based on the simple Jones matrix model, discussed more recently in Ref. [15]. It was used to understand basics of polarization dynamics inside spin-VCSELs at steady-state and to interpret the first experimental approaches to quantify and compensate the birefringence $[16,17]$. More extensive formalism has been developed in the framework of $4 \times 4$ transfer [18] and scattering-matrix formalism [19], built upon the ideas from Refs. [20, 21]. This layer-by-layer approach has been proven to be able to explain experimental results in great details, considering local optical properties such as linear and circular anisotropies i) at the interfaces of III-V semiconductors, ii) at the surface and iii) originating from spin injector and circular dichroism due to spin imbalance in QWs. It is largely useful also for detailed experimental studies of spin-VCSEL anisotropies, as recently shown [22] and eventually for implementing advanced theoretical models of anisotropic QW gain [23- 
25]. From the perspective of ultrafast dynamics, the integration of spin-VCSELs with highly-birefringent photonic crystal mirrors (spin-PCSELs) is very promising. The results in Ref. [26] shows, that the scattering matrix formalism manifests as one of the most relevant technique to model such devices. All of the mentioned frameworks are limited to near threshold operation. This could be eventually overcome by the above-threshold generalization of the matrix formalism or using the steady-state $a b$ initio lasing theory (SALT) algorithm applied to spinVCSELs [27-29].

On the other hand, the tools for temporal modeling of spin-VCSELs are mainly represented by the spin-flip model (SFM), as the simplified Maxwell-Bloch equations $[30,31]$, derived in a more intuitive way independently by Travagnin [32], developed originally for the conventional VCSELs. The SFM considers time evolution of circularly-polarized field components in the presence of linear birefringence and linear dichroism introduced phenomenologically, neglecting any linear gain anisotropies. Later, also the case of possibly misaligned axes of birefringence and dichroism has been considered [33, 34], motivated by the experimental techniques to induce such phenomena [35]. This was generalized in order to include the possible frequency variation of QW gain and to eventually explain the polarization switching [36]. A true disadvantage of SFM is that it can not describe selfconsistently the geometric complexity of VCSEL cavity and localized nature of anisotropies and the dynamicsrelated parameters must be obtained from experiments. This was solved by the robust spatio-temporal models for VCSELs [37, 38]. Such models used the shapes of vectorial eigenmodes obtained by solving the cold-cavity problem, taking into account the lateral dimensions of devices and carrier diffusion. More recently, the indexguiding effects and transverse mode dynamics has been studied in Refs. [39, 40] using generalized SFM with anisotropy rates extracted from theory, but for a simple effective structure. The extensive spatio-temporal modeling of VCSELs is described in an unified way in Refs. [41, 42]. Recently, the general coupled mode theory has been derived to describe laterally coupled spinVCSELs [43], which shares certain similarity with our present work. Particular attention should be paid to the laser theory developed originally for coupled cavity lasers [44], built upon the pioneering work of Haken, Sargent and Lamb [45, 46]. More recently, it was used to describe the micro-cavity lasers, finding excellent agreement with finite-difference time-domain (FDTD) simulations [47]. The approach has been generalized also to describe lateral effect in half-VCSELs [48, 49], however, neglecting the detrimental effects of anisotropies and spin pumping.

In this paper, we develop a self-consistent formalism for modeling of steady-state and time-dependent emission of spin-VCSELs with large local anisotropies. We generalize robust matrix formalism, based on our previous work [19]. Moreover, we extend the coupled mode theory of Hodges et al. [44]. The model respects the spatial variation of electric field and its vector nature, together with local anisotropies within the cavity. Additionally, we propose and design the spin-VCSEL structure with intra-cavity grating. We demonstrate that it is possible to reach $\mathrm{THz}$ frequency splitting between modes, which paves the way for important advancements in $\mathrm{THz}$ photonics.

This paper is organized as follows. In Sec. II, the semiclassical optical Bloch equations for spin-VCSEL in the 2-level approximation are derived. We consider possible linear anisotropies in the passive cavity as well as inside QWs, in contrary to most approaches up to date. In Sec. III, we generalize the matrix formalism. Instead of using active dipolar layers to describe QW gain, we derive effective susceptibility of spin-polarized QWs. Consequently, entire structures can be described using Yeh's formalism in an unified way. Sec. IV contains the generalized coupled mode theory of spin-VCSEL, derived for circularly-polarized vectorial eigenmodes. Alternative bases are discussed. Our approach allows to treat self-consistently entire spin-VCSEL structure, including all local anisotropies, leading for example to simple analytic expressions for anisotropy rates. In a certain sense, it can be considered as the time-dependent counter-part to SALT, as noted in Ref. [47]. Motivated by our previous results [50], the theory naturally reduces to extended SFM, which is derived in Sec. V. The extended SFM describes also the linear gain anisotropy, which is often neglected. Finally, in Sec. VI, we apply developed formalism to design and optimize the performance of realistic spin-VCSEL with intra-cavity birefringent grating with large potential for $\mathrm{THz}$ photonics applications, such as ultrafast data transfer and generation of coherent $\mathrm{THz}$ radiation. Appendix A and Appendix B are devoted to theory of transfer and scattering matrices and calculation of cavity decay rate, respectively.

\section{SEMI-CLASSICAL DESCRIPTION OF SPIN-VCSEL}

\section{A. General approach and approximations}

Let us now describe the broad picture of how we approach the problem of the modeling of spin-VCSELs. We use the modified semi-classical spatio-temporal formalism of Maxwell-Bloch equations, based on combination of quantum-mechanical density matrix and classical Maxwell's theory of electrodynamics.

Concerning the QW gain media, it is modeled by an ensemble of 2-level quantum systems distributed inside active layers. Electron conduction band states (excited) and heavy-hole states in valence band (ground) are coupled via electric dipole interaction, as shown in Fig. 1(a). Each of these levels posses two spin sub-levels, which can be mixed due to relaxation processes. By spin state, we understand its projection along $\boldsymbol{z}$-axis. The mixing between heavy-hole spin states can be considered much 


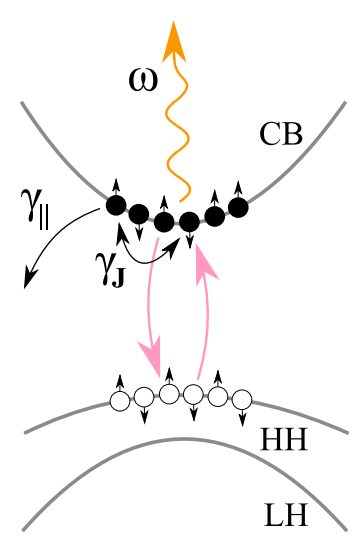

(a)

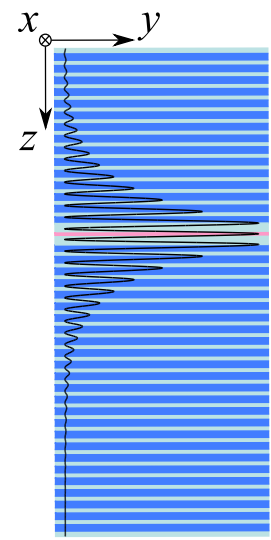

(b)
FIG. 1. (a) Approximate band structure inside semiconductor QW with spin degree of freedom. It is assumed, that radiative re-combinations occur only between conduction band (CB) electrons and heavy-hole $(\mathrm{HH})$ states near $\Gamma$-point. (b) SpinVCSEL structure considered here, in which only the propagation of electromagnetic waves parallel to $\boldsymbol{z}$-axis is considered.

faster than in case of electrons in the conduction band. The energy difference between excited and ground states near the $\Gamma$-point $\Delta E=\hbar \omega_{0}$ is close to the energy of laser field photons $\hbar \omega$. Thus, any optical coupling to lighthole states is neglected due to HH-LH (heavy hole - light hole) energy splitting in the QW potential.

We consider only paraxial (with respect to $\boldsymbol{z}$-axis) wave propagation in the multilayer laser cavity as depicted in Fig. 1(b). In this approximation, the structure is treated layer-by-layer, which has never been considered among the time-dependent models of spin-VCSELs. Thus, our approach is sensitive to any small changes in the local optical and geometrical properties of any layer, providing a more realistic description of spin-VCSELs. Opticalmaterial properties of each layer of the structure are described by the permittivity tensor. Such layer-by-layer strategy for studying spin-VCSELs, with particular focus on optical anisotropies, has been already demonstrated in our earlier contributions $[18,19,22]$. Here, we extend our approach also to time-domain simulations. We study the structures with linear birefringence and linear dichroism in the passive layers, and additionally with the linear gain anisotropy inside active layer, which is often neglected.

\section{B. Optical-material properties}

\section{Linear birefringence and dichroism inside cavity}

Since our aim is to develop the polarization-sensitive formalism, let us shortly mention the typical anisotropies located inside the passive parts of laser cavity, together with their physical origin. Apart from active layer anisotropies, which may depend on the gain, spin-
VCSEL structures are strongly impacted by the linear birefringence, and the consequent linear dichroism, in the background semiconductor media. According to detailed theoretical and experimental investigations, it is mostly due to combined effects of strain in the structures [51] and the phenomena at semiconductor-semiconductor and semiconductor-air interfaces, in which the crystallographic symmetry can be reduced [52]. The strain can be of native origin, caused for example by the lattice mismatch of crystal media in neighboring layers, or induced externally by heating or simple mechanical techniques [35]. Another source of anisotropy may originate from the crystal relaxation in the layer at the top of the VCSEL. Overall anisotropy is enhanced additionally by the electro-optically active media due to the applied static electric field [53].

\section{Semiconductor $Q W$ with linear gain anisotropy}

Let us now consider the spin-polarized semiconductor QW with linear gain anisotropy. We are particularly interested in the part of optical response of semiconductor $\mathrm{QW}$, that originates from the pumped laser transitions in the presence of linear gain anisotropy. In the absence of such anisotropy, radiative electron-hole re-combinations in the two spin channels with opposite electron spin projections $\uparrow, \downarrow$ give rise to circularly polarized fields. We will use standard procedure to derive the susceptibility using dipole matrix elements.

Let us consider the electric dipole approximation, in which the general quantum dipole matrix element is given by $[45,54]$ :

$$
\boldsymbol{\theta}_{i \rightarrow f}=\int \psi_{f}(\boldsymbol{r}) \hat{\boldsymbol{\theta}} \psi_{i}(\boldsymbol{r}) \mathrm{d}^{3} \boldsymbol{r},
$$

where $\boldsymbol{\theta}_{i \rightarrow f}$ is the electric dipole operator matrix element between states $|i\rangle$ and $|f\rangle, \hat{\boldsymbol{\theta}}=\left[\hat{\theta}_{x}, \hat{\theta}_{y}, \hat{\theta}_{z}\right]^{T}$ is the vector of electric dipole operators, $\psi_{i}(\boldsymbol{r})$ and $\psi_{f}(\boldsymbol{r})$ are spatial parts of wave-functions of coupled initial and final states, respectively.

In the case of semiconductor QW with quantization axis parallel to $\boldsymbol{z}$, the dipole matrix elements for two spin channels can be generally written as [24]:

$$
\begin{aligned}
& \boldsymbol{\theta}_{\uparrow} \propto \theta\left(\boldsymbol{e}_{+}+\beta \boldsymbol{e}_{-}\right), \\
& \boldsymbol{\theta}_{\downarrow} \propto \theta\left(\boldsymbol{e}_{-}+\beta \boldsymbol{e}_{+}\right),
\end{aligned}
$$

where $\boldsymbol{e}_{ \pm}$are Jones vectors describing polarization state of circularly polarized optical fields, $\theta$ is a certain average dipole matrix element and $\beta$ is the general parameter, which describes the linear gain anisotropy under consideration. In the case of strained QW, with principal axes along [110] and [110] crystallographic directions, $\beta$ can be considered as a band-mixing parameter. Using such interpretation, we can write $\beta=-i \mathcal{D}$ where $\mathcal{D}$ is proportional to the difference of applied or internal stress or equivalent effect along the principal axes: $\sigma_{[110]}-\sigma_{[1 \overline{1} 0]}$. 
Dipole matrix elements of both spin channels $\boldsymbol{\theta}_{\uparrow, \downarrow}$ can be expressed using normalized Jones vectors as $\boldsymbol{\theta}_{\uparrow, \downarrow}=$ $\theta \boldsymbol{e}_{\uparrow, \downarrow}$, which are derived to be:

$$
e_{\uparrow}=\left(e_{\downarrow}\right)^{*}=\frac{1}{\sqrt{2\left(1+\mathcal{D}^{2}\right)}}\left[\begin{array}{c}
1-i \mathcal{D} \\
-i+\mathcal{D}
\end{array}\right]
$$

Note that the present expression reduces to purely circular eigenmodes $\left(\boldsymbol{e}_{ \pm}\right)$in absence of any linear anisotropy, giving the standard result obtained using unperturbed electronic wave-functions.

Similar results with however a different sign convention have been obtained using the approach of Fördös et al. [19]: by defining relation between optical transition matrix elements. Using their mathematical notation, linear gain anisotropy could be introduced in the following way: $\Pi_{[110]}=[1+(1-\Delta) / 2] \Pi_{0}, \Pi_{[1 \overline{1} 0]}=[1-(1-\Delta) / 2] \Pi_{0}$, where $\Pi_{[110],[1 \overline{1} 0]}$ stand for transition matrix elements along anisotropy principal axes and $\Pi_{0}$ is the average matrix element. It can be shown, that $\mathcal{D}=(1-\Delta) / 2$.

It appears useful to define the dimensionless susceptibilities $\hat{\mathcal{T}}_{\uparrow, \downarrow}$ of particular spin channels using their respective Jones vectors [54]:

$$
\hat{\mathcal{T}}_{\uparrow, \downarrow}=\boldsymbol{e}_{\uparrow, \downarrow} \otimes \boldsymbol{e}_{\uparrow, \downarrow}^{\dagger} .
$$

Under pumping, induced dipole moment densities $\tilde{\boldsymbol{P}}_{\uparrow, \downarrow}$ are proportional to $\hat{\mathcal{T}_{\uparrow, \downarrow}} \boldsymbol{E}$ in the steady-state regime.

It should be noted, that since now, for the sakes of simplicity, all of the calculations will be performed in the basis spanned by the crystallographic axes [110] and [110]. By this, we mean, that electric field vector will have following components $\boldsymbol{E}=\left[E_{[110]}, E_{[1 \overline{1} 0]}\right]^{T}$. In order to obtain correct Jones vectors and susceptibilities, one should perform a rotation of reference frame around $\boldsymbol{z}$ by angle of $45^{\circ}$. Equivalently, we could just take $\beta=\mathcal{D}$. In the given basis, the dimensionless susceptibilities are

$$
\hat{\mathcal{T}}_{\uparrow}=\left(\hat{\mathcal{T}}_{\downarrow}\right)^{*}=\frac{1}{2\left(1+\mathcal{D}^{2}\right)}\left[\begin{array}{cc}
(1+\mathcal{D})^{2} & i\left(1-\mathcal{D}^{2}\right) \\
-i\left(1-\mathcal{D}^{2}\right) & (1-\mathcal{D})^{2}
\end{array}\right] .
$$

One can see, that the dipole interactions of the electromagnetic fields with specific spin channel is different for waves polarized along [110] or [11̄0] directions, until $\mathcal{D}=0$.

\section{Maxwell-Bloch equations}

Our approach to model the spatio-temporal dynamics of anisotropic spin-lasers is based on modified MaxwellBloch equations. The modification is based on the fact, that we need to describe anisotropic laser cavity and the gain media. As we already noted, each spin channel in semiconductor QW is modeled by an ensemble of 2-level systems. Time evolution of an open 2-level system can be described by a Liouville - von Neumann equation for the density matrix:

$$
i \hbar \frac{\partial}{\partial t} \hat{\rho}_{\mu}=\left[\hat{\mathcal{H}}_{0, \mu}+\hat{\mathcal{H}}_{d, \mu}, \hat{\rho}_{\mu}\right]
$$

where $\mu=\uparrow, \downarrow$. The operator $\hat{\mathcal{H}}_{0, \mu}$ stands for the unperturbed diagonalized Hamiltonian (in the sense of lightmatter interaction):

$$
\hat{\mathcal{H}}_{0, \mu}=\frac{1}{2}\left[\left(E_{g, \mu}+E_{e, \mu}\right) \hat{1}+\left(E_{g, \mu}-E_{e, \mu}\right) \hat{\sigma}_{z}\right]
$$

where $E_{g / e, \mu}$ are energies of the ground and excited states at spin channel $\mu$. Operators $\hat{1}$ and $\hat{\sigma}_{z}$ stand for the unity operator and the diagonal Pauli matrix, respectively.

The ground and excited states are coupled to the electric field via the electric dipole interaction described by the operator:

$$
\hat{\mathcal{H}}_{d, \mu}=-\hat{\boldsymbol{\theta}}_{\mu} \cdot[\boldsymbol{E}(\boldsymbol{r}, t) \exp (i \omega t)+\text { c.c. }] \text {, }
$$

where $\hat{\boldsymbol{\theta}}_{\mu}$ is the electric dipole operator evaluated at the $\mu$-th spin channel, $\boldsymbol{E}$ is the positive-frequency component of oscillating electric field and $\omega$ is the central frequency of the cavity laser field.

Equations of motion for the density operator are combined with the classical electric field wave equation, where we assume, that the dipole moment density responsible for the lasing process [defined as $N_{0, \mu} \operatorname{Tr}\left(\hat{\rho}_{\mu} \hat{\boldsymbol{\theta}}_{\mu}\right)=\tilde{\boldsymbol{P}}_{\mu}(\boldsymbol{r}, t) \exp (i \omega t)+$ c.c., where $N_{0, \mu}$ is the concentration of idealized 2-level systems] represents the source term in the wave equation. We then derive the modified Maxwell-Bloch equations, describing the spatiotemporal evolution of the dipole moment densities $\tilde{\boldsymbol{P}}_{\mu}$, spin carrier concentrations $N_{\mu}$ and intra-cavity electric field $\boldsymbol{E}$ according to [45]:

$$
\begin{gathered}
\frac{\partial}{\partial t} \tilde{\boldsymbol{P}}_{\mu}(\boldsymbol{r}, t)=-\left(\gamma_{\perp}+i \delta_{\mu}\right) \tilde{\boldsymbol{P}}_{\mu}(\boldsymbol{r}, t)+\frac{i}{\hbar}|\theta|^{2} N_{\mu}(\boldsymbol{r}, t) \hat{\mathcal{T}}_{\mu}(\boldsymbol{r}) \boldsymbol{E}(\boldsymbol{r}, t), \\
\frac{\partial}{\partial t} N_{\mu}(\boldsymbol{r}, t)=\Lambda_{\mu}(\boldsymbol{r}, t)-\gamma_{\|} N_{\mu}(\boldsymbol{r}, t)-\gamma_{J}\left[N_{\mu}(\boldsymbol{r}, t)-N_{\mu^{\prime}}(\boldsymbol{r}, t)\right]+\frac{2 i}{\hbar}\left[\boldsymbol{E}^{\dagger}(\boldsymbol{r}, t) \tilde{\boldsymbol{P}}_{\mu}(\boldsymbol{r}, t)-\tilde{\boldsymbol{P}}_{\mu}^{\dagger}(\boldsymbol{r}, t) \boldsymbol{E}(\boldsymbol{r}, t)\right], \\
{\left[c^{2} \nabla^{2}-\hat{\varepsilon}_{m}(\boldsymbol{r}) \frac{\partial^{2}}{\partial t^{2}}-\tilde{\kappa} \frac{\partial}{\partial t}\right] \boldsymbol{E}(\boldsymbol{r}, t) \exp (i \omega t)=\frac{1}{\varepsilon_{0}} \frac{\partial^{2}}{\partial t^{2}} \sum_{\mu=\uparrow, \downarrow} \tilde{\boldsymbol{P}}_{\mu}(\boldsymbol{r}, t) \exp (i \omega t),}
\end{gathered}
$$


density matrix coherences $\gamma_{\perp}$, spin-carrier pumping rate $\Lambda_{\mu}$, decay coefficient $\gamma_{\|}$and spin-mixing rate $\gamma_{J}$. The characteristic decay of the electric field is described by $\tilde{\kappa}$, which is specific for given design of optical cavity and its use is well-justified by Poynting theorem in Appendix B.

Apart from possible gain anisotropies in the QWs, which are described self-consistently by $\hat{\mathcal{T}}_{\mu}$, it is possible to include any local cavity anisotropies via relative permittivity tensor $\hat{\varepsilon}_{m}$ of the background semiconductor media, which differs from layer to layer.

In order to provide a complete spatio-temporal solution to these partial differential equations, we have chosen not to consider any brute-force time-consuming techniques. Instead of this, we use transfer and scattering matrix formalism to describe the steady-state properties of lasing of spin-VCSELs and the approach based on the coupled mode theory to investigate the time-evolution of optical fields inside spin-VCSELs.

\section{STEADY-STATE LASER EMISSION: MATRIX DESCRIPTION}

This section introduces alternative, and more general, description of multilayer laser structures, such as spinVCSELs, based on transfer and scattering matrix formalism, first proposed in Ref. [19]. Similarly as here, each layer in the structure has been described separately. However, in contrary to our modified approach, active layers required a special treatment, since they were approximated as infinitesimally thin active dipolar layers. Such approximation is not necessary here, which provides several advantages. The use of active dipolar layer approximation is well justified for resonant structures, but the lasing threshold is not determined correctly, if one changes the position within the cavity field profile. This is solved self-consistently here. Entire laser structure, together with amplifying active media (QWs/QDs), is treated in a straightforward way, using standard Yeh's procedure for solving electromagnetic wave propagation inside an arbitrary layered structures [55]. Using active dipolar layer approximation, the implementation of the formalism for multiple-QW structures becomes difficult. Susceptibility approach is more suitable for incorporating realistic gain spectra of QWs, as well as possible field saturation effects.

\section{A. Light amplification in spin-polarized anisotropic media}

\section{Derivation of susceptibility}

For the purposes of matrix formalism, we derive the electric susceptibility (and later permittivity) of active media using our modified Maxwell-Bloch equations. Due to the additivity of electric dipole moments, we can write the total dipole moment density of gain media as
$\boldsymbol{P}=\boldsymbol{P}_{m}+\tilde{\boldsymbol{P}}$, where $\boldsymbol{P}_{m}$ is the background contribution, originating from the optical transitions except the lasing ones, which is given by $\tilde{\boldsymbol{P}}$. The contribution of lasing transition in the steady-state operation regime of the laser is:

$$
\tilde{\boldsymbol{P}}=\sum_{\mu=\uparrow, \downarrow} \tilde{\boldsymbol{P}}_{\mu}=\varepsilon_{0} \hat{\chi}_{a} \boldsymbol{E}
$$

where $\hat{\chi}_{a}$ stands for susceptibility of active media. Assuming $\delta_{\uparrow}=\delta_{\downarrow}=\delta$ and steady-state laser oscillation, Eq. (9) leads to:

$$
\tilde{\boldsymbol{P}}=\sum_{\mu=\uparrow, \downarrow} i \frac{1}{\gamma_{\perp}+i \delta} \frac{|\theta|^{2}}{\hbar} N_{\mu} \hat{\mathcal{T}}_{\mu} \boldsymbol{E} .
$$

Comparing this result with Eq. (12), we can derive:

$$
\hat{\chi}_{a}=i \frac{1}{\gamma_{\perp}+i \delta} \frac{|\theta|^{2}}{\hbar \varepsilon_{0}}\left(N_{\uparrow} \hat{\mathcal{T}}_{\uparrow}+N_{\downarrow} \hat{\mathcal{T}}_{\downarrow}\right) .
$$

Because we know, that optical response of QW is not described by Lorentz line-shape, we use the approximate relation between frequency detuning $\delta$ and inverse coherence lifetime $\gamma_{\perp}: \alpha=\delta / \gamma_{\perp}$, where $\alpha$ is the linewidth enhancement factor. Additionally, the steady-state values of spin carrier concentrations $N_{\uparrow, \downarrow}$ can be straightforwardly calculated using Eq. (10). Note, that $N_{\uparrow, \downarrow}$ are generally the functions of electric field, which leads to nonlinear problem. However, this is out of the scope of present work, since we are mainly interested in the nearthreshold steady-state oscillation. Consequently, the expression for the susceptibility contribution responsible for light amplification $\hat{\chi}_{a}$ can be written in the compact form as:

$$
\hat{\chi}_{a}=i \bar{\chi}(1-i \alpha) \hat{\mathcal{T}},
$$

where the definition of optical gain tensor $\hat{\mathcal{T}}$ from Ref. [19] $\left(N_{\uparrow}+N_{\downarrow}\right) \hat{\mathcal{T}}=N_{\uparrow} \mathcal{\mathcal { T }}_{\uparrow}+N_{\downarrow} \hat{\mathcal{T}}_{\downarrow}$ has been used:

$$
\hat{\mathcal{T}}=\frac{1}{2\left(1+\mathcal{D}^{2}\right)}\left[\begin{array}{cc}
(1+\mathcal{D})^{2} & i\left(1-\mathcal{D}^{2}\right) \mathcal{P}_{s} \\
-i\left(1-\mathcal{D}^{2}\right) \mathcal{P}_{s} & (1-\mathcal{D})^{2}
\end{array}\right],
$$

in which the effective degree of electron spin polarization $\mathcal{P}_{s}$ has been introduced. According to Eq. (10), when $\boldsymbol{E} \rightarrow 0$ (at threshold), we can write:

$$
\mathcal{P}_{s}=\frac{\gamma_{\|}}{\gamma_{\|}+2 \gamma_{J}} \mathcal{P}_{J},
$$

where $\mathcal{P}_{J}$ is the pump spin polarization. The fact, that pumping rates $\Lambda_{\uparrow, \downarrow}$ can be expressed using unsaturated carrier concentrations $N_{0 \uparrow, \downarrow}$, has been used. The scalar part $\bar{\chi}$ is defined as:

$$
\bar{\chi}=\frac{1}{\left(1+\alpha^{2}\right) \gamma_{\perp}} \frac{|\theta|^{2}}{\hbar \varepsilon_{0}}\left(N_{0 \uparrow}+N_{0 \downarrow}\right) .
$$

The specific line shape (Lorentz in this case) is not considered, because $\bar{\chi}$ is controlled quantity here as a certain 
normalized pump rate, or the quantity to be found in the case of threshold condition. In this way, we model relatively broad gain profile of semiconductor QW. This can be further generalized to describe more precisely the dependence on carrier concentration and photon frequency, by going beyond 2-level gain media approximation.

The total electric susceptiblity consists of background part $\hat{\chi}_{m}$ and active part $\hat{\chi}_{a}$, which is generally timedependent due to carrier dynamics:

$$
\hat{\chi}(\boldsymbol{r}, t)=\hat{\chi}_{m}(\boldsymbol{r})+\hat{\chi}_{a}(\boldsymbol{r}, t) .
$$

As one can expect, this time-dependence is neglected in the present section.

\section{Wave equation in the active media}

In order to derive the total matrix of the system one considers, we solve the wave equation in each layer separately. Since most of the layers in the laser system are optically isotropic or with weak anisotropies, we are primarily interested in solving the wave equation in the active layers. The propagation constants and polarization eigenmodes are derived.

The wave equation (11) reduces in the steady-state to:

$$
\left[c^{2} \nabla^{2}-\hat{\varepsilon}(\boldsymbol{r}) \frac{\partial^{2}}{\partial t^{2}}\right] \boldsymbol{E}(\boldsymbol{r}) \exp (i \omega t)=0,
$$

where we assume, that any decay processes in the cavity described by $\kappa$ are self-consistently included by the transfer matrix formalism. We introduced total relative permittivity tensor $\hat{\varepsilon}(\boldsymbol{r})=\hat{1}+\hat{\chi}_{m}(\boldsymbol{r})+\hat{\chi}_{a}(\boldsymbol{r})$. The spatial variation of the field inside active layer can be described by the plane waves: $\boldsymbol{E}(\boldsymbol{r})=\boldsymbol{E}_{0} \exp (-i \boldsymbol{q} \boldsymbol{r})$, where $\boldsymbol{E}_{0}$ is the amplitude of the electric field inside active layer. As we know, differential operators from the wave equation act as follows: $\boldsymbol{\nabla} \rightarrow-i \boldsymbol{q}$ and $\partial_{t} \rightarrow i \omega$. Omitting the spatial the spatial variation of permittivity, since we analyze single layer, we obtain the Helmholtz equation:

$$
\left[\hat{\varepsilon}\left(\frac{\omega}{c}\right)^{2}-\boldsymbol{q}^{2}\right] \boldsymbol{E}_{0}=0,
$$

Two assumptions are used here. First, only the propagation parallel to $\boldsymbol{z}$ is considered, so the normalized propagation constant is defined $\tilde{q}=|\boldsymbol{q}| /(\omega / c)$, which stands for $z$-component of the wave-vector, normalized to the vacuum wave-number. Second, we use the isotropic background media $\hat{\varepsilon}_{m}=\varepsilon_{Q W} \hat{1}$. The possible generalization for anisotropic background semiconductor media is straightforward and does not rise any difficulty. Using Eqs. (15) and (21), and approximating the term $1 /\left[2\left(1+\mathcal{D}^{2}\right)\right]$ using $1 / 2$, since $\mathcal{D}^{2}$ is typically very small $\left(\mathcal{D}^{2}<<1\right)$, the normalized propagation constants inside active layers are derived:

$$
\begin{aligned}
\tilde{q}_{1,2}^{2}= & \varepsilon_{Q W}+i \frac{\bar{\chi}}{2}(1-i \alpha)\left(1+\mathcal{D}^{2}\right) \\
& \pm i \frac{\bar{\chi}}{2}(1-i \alpha) \sqrt{(2 \mathcal{D})^{2}+\left[\left(1-\mathcal{D}^{2}\right) \mathcal{P}_{s}\right]^{2}}
\end{aligned}
$$
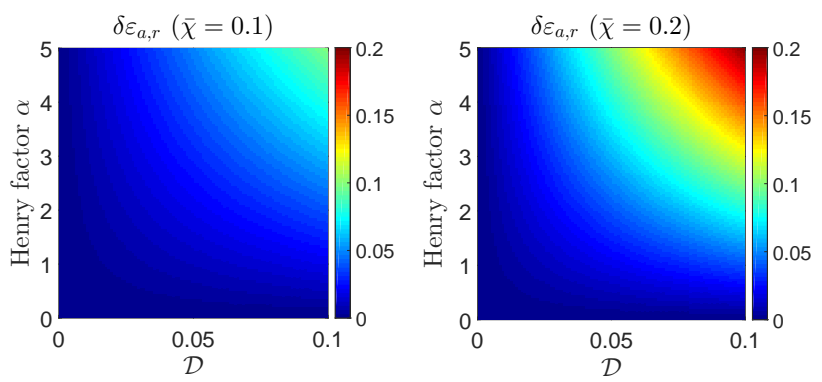

FIG. 2. Birefringence $\delta \varepsilon_{a, r}$ induced by phase-amplitude coupling as a function of the gain dichroism $\mathcal{D}$ and the Henry factor $\alpha$.

Note, that due to anisotropies, this result contains 4 possible propagation constants, describing 2 forwardpropagating and 2 backward-propagating waves, which build up the resulting standing wave. The eigenmode polarizations $\boldsymbol{e}_{j}$, defined as $\boldsymbol{E}_{0 j}=E_{0 j} \boldsymbol{e}_{j}$, are:

$$
\boldsymbol{e}_{1,2} \propto\left[\begin{array}{c}
1 \\
i \frac{2 \mathcal{D} \mp \sqrt{(2 \mathcal{D})^{2}+\left[\left(1-\mathcal{D}^{2}\right) \mathcal{P}_{s}\right]^{2}}}{\left(1-\mathcal{D}^{2}\right) \mathcal{P}_{s}}
\end{array}\right] .
$$

\section{Phase-amplitude coupling}

Although the anisotropy parameter $\mathcal{D}$ has been introduced in order to quantify the linear gain anisotropy, its non-zero value has also consequences for phase anisotropies. It is well-known, that due to phaseamplitude coupling mechanisms, the gain anisotropy may contribute to the birefringence of the media. Let's assume for example strained quantum well with $\mathcal{P}_{s}=0$. In our convention, the permittivity tensor of such media would be diagonal with different values for waves polarized along [110] and [1̄ㅣ] directions:

$$
\begin{aligned}
& \varepsilon_{a, x x}=\varepsilon_{Q W}+i \frac{\bar{\chi}}{2}(1-i \alpha)(1+\mathcal{D})^{2}, \\
& \varepsilon_{a, y y}=\varepsilon_{Q W}+i \frac{\bar{\chi}}{2}(1-i \alpha)(1-\mathcal{D})^{2} .
\end{aligned}
$$

Induced linear birefringence can be quantified using the difference of real parts of $\varepsilon_{x x}$ and $\varepsilon_{y y}$. The calculation yields:

$$
\delta \varepsilon_{a, r}=\operatorname{Re}\left\{\varepsilon_{a, x x}-\varepsilon_{a, y y}\right\}=2 \bar{\chi} \alpha \mathcal{D} .
$$

Assuming typical values $\bar{\chi}=0.1, \alpha=3$ and $\mathcal{D}=0.025$ (or equivalently $1-\Delta \cong 0.95[19]$ ), one obtains $\delta \varepsilon_{a, r}=$ 0.015 . This can have significant effects on the polarization dynamics of spin-VCSEL structures. Note, that with $\alpha=0$ there is no effective birefringence due to linear gain anisotropy, as shown in Fig. 2. 


\section{B. The resonance condition}

The threshold modes of spin-VCSEL structure are used in the Sec. IV as a basis for the time-dependent coupled mode theory. Exact spatial distribution of threshold modes is used to evaluate overlap integrals, which describe effects of perturbations in optical cavity, such as optical anisotropies. In the following, the procedure to extract of such modes is described.

The eigenmodes extraction is demonstrated on a model multiple-QW structure as depicted in FIG. 3. According to Appendix A, the characteristic matrix of general $n$-th layer is $\mathbf{T}^{(n)}=\mathbf{D}^{(n)} \mathbf{P}^{(n)}\left[\mathbf{D}^{(n)}\right]^{-1}$, where $\mathbf{D}^{(n)}$ is dynamic matrix, which is used to calculate the components of electromagnetic field using amplitudes of particular running waves. The propagation and the amplification of running waves in the media is given by $\mathbf{P}^{(n)}$. The notation $\mathbf{T}_{a}^{(n)}$ stands for the characteristic matrix of $n$-th active layer. According to Eq. (A6), the total matrix $\mathbf{M}$ is:

$$
\mathbf{M}=\left[\mathbf{D}^{(0)}\right]^{-1} \mathbf{T}^{(1)} \cdots \mathbf{T}_{a}^{(1)} \cdots \mathbf{T}^{(N)} \mathbf{D}^{(N+1)}
$$

Note, that $\mathbf{M}=\mathbf{M}(\lambda, \bar{\chi})$, where the wavelength $\lambda$ and scalar part of active layer susceptibility $\bar{\chi}$, which is proportional to the pump rate, are the quantities to be found. Their threshold values can be found by satisfying the waveguiding condition:

$$
\mathbf{M}_{11} \mathbf{M}_{33}-\mathbf{M}_{13} \mathbf{M}_{31}=0 .
$$

Alternatively, the scattering matrix $\mathbf{S}$ of the system can be calculated, allowing more straightforward calculation of the amplitudes $\boldsymbol{A}_{\text {out }}$ of emitted waves:

$$
\mathbf{S}^{-1} \boldsymbol{A}_{\text {out }}=0 .
$$

One can see, that the condition for non-trivial solution is

$$
\operatorname{det}\left(\mathbf{S}^{-1}\right)=[\operatorname{det}(\mathbf{S})]^{-1}=0,
$$

which is equivalent to the standard waveguiding condition in the transfer matrix picture [see Eq. (27)].

\section{TEMPORAL COUPLED MODE THEORY}

\section{A. The basis}

\section{The basis of circularly-polarized fields}

In this section, we develop and describe the coupled mode theory of spin-VCSELs based on the MaxwellBloch equations derived in Sec. II C. We have adopted and generalized the approach first introduced in Ref. [44] and more recently in Ref. [47]. Consequently, our derivations are structured according to these papers. The approach is based on the projection of the Maxwell-Bloch

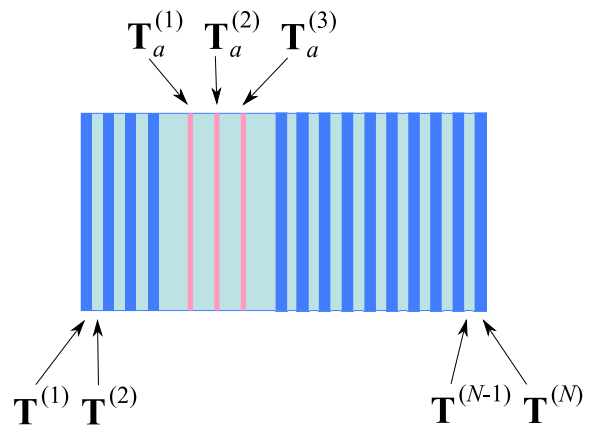

FIG. 3. Scheme of a muliple-QW spin-VCSEL device, consisting of $N+3$ layers. Each layer is described by its own permittivity tensor $\hat{\varepsilon}^{(n)}$ and thickness $d^{(n)}$.

equations onto a certain basis, which is specific for each cavity. The novelty introduced here is, that the vector nature of the basis functions is respected. Additionally to this, our basis functions are calculated for the active device, in contrary to the most of works $[37,40]$. It allows to include self-consistently all of the polarization-dependent properties of cavity.

There are more possible options for choosing a correct basis, which will be discussed later. In this work, we derive the coupled mode equations using the basis of circularly-polarized fields, that can be obtained from our modified matrix approach for the extraction of the laser eigenmodes. We comment on several reasons, which are mostly practical, why such basis is the appropriate one:

i) The first reason lies in the optical selection rules and in the fact, that particular radiative electron-hole re-combinations in device of spin-VCSEL geometry generates circularly polarized photons. In this sense, the anisotropies within the cavity can be considered as a perturbations to those circular basis functions.

ii) The second reason for using the circular basis is based on its mathematical properties. We know, that the positive-frequency component of electric field can be generally decomposed into:

$$
|E\rangle=\sum_{k} E_{k}|k\rangle \exp \left(i \delta \omega_{k} t\right)
$$

where we have used the compact bra-ket notation to avoid the tedious integral equations in the next parts of manuscript. $E_{k}$ stands for the time-dependent amplitude of eigenmode (or basis function, alternatively) $|k\rangle$ and $\delta \omega_{k}$ is the frequency shift of given mode with respect to the certain central frequency $\omega$ (see Sec. II C) of laser field oscillations. The circular basis can be considered as degenerated from the frequency point of view and thus $\delta \omega_{k} \rightarrow 0$. The total field can be decomposed as a sum $|E\rangle=E_{+}|+\rangle+E_{-}|-\rangle$in this case, which significantly simplifies the mathematical derivations. Next practical advantage is the orthogonality of circular basis $\langle+\mid-\rangle=0$.

iii) The last reason is, that we would like to show the connection to some previous work in this field. Namely, 


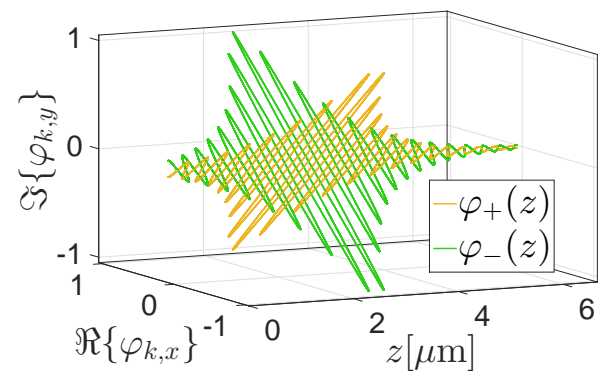

FIG. 4. The spatial distribution of the basis functions $\boldsymbol{\varphi}_{k}(z)=\langle z \mid k\rangle$ within the laser cavity.

to provide the rigorous background to well-known SFM as well as its extension.

Typical shapes of the basis functions $\varphi_{ \pm}(z)=\langle z \mid \pm\rangle$ calculated numerically are shown in the Fig. 4 as the functions of position within the cavity.

\section{Other basis options}

Generally, the choice of a particular basis depends on the properties of the laser structure. For example, in our polarization-focused approach, one can estimate which element of the laser cavity (such as spin-polarized QWs, or birefringent layers in the Bragg reflectors) is the most dominant in determining laser's performance.

Consequently, in the case of a structure with very strong optical anisotropies, such as strained VCSELs studied in Ref. [13], it would be more appropriate to choose the linearly polarized basis functions. There are two options, how to apply the linear basis. First, one can use the orthogonal linear functions with degenerate frequencies. Any anisotropies, such as the linear birefringence or the gain anisotropy originating from electron spin imbalance in active regions play the role of perturbations. Another option is to use linear basis functions of different frequency, calculated using already anisotropic laser cavity. However, it would lead to mathematical difficulties.

Alternatively, it is possible to construct the coupled mode theory for the real eigenmodes, which do not have to be orthogonal. This is the most general approach, because it considers all of the optical properties of the given structure at the same time, without treating some of them as a perturbation. The ansatz for the electric field looks slightly different in this case (idea is based on the work in Ref. [43]):

$$
\boldsymbol{E}(z, t)=\sum_{k} \boldsymbol{E}_{k}(t) \varphi_{k}(z) \exp \left(i \delta \omega_{k} t\right)
$$

We use a standard notation here, to emphasize, that in this case the time-dependent amplitudes $\boldsymbol{E}_{k}$ contains the polarization vector and we consider only scalar spatiallyvarying basis functions $\varphi_{k}$. The approximation here is, that $\varphi_{k}$ is time-independent, which only introduces a small inaccuracy in the formalism.

\section{B. Derivation of the coupled mode equations: structures of arbitrary geometry}

\section{Dipole moment density decomposition}

The first step in deriving the rate equations consists of expressing the dipole moment densities $\tilde{\boldsymbol{P}}$ in the considered circular basis. We use the compact bra-ket notation, thus, the Eq. (9) becomes:

$$
\frac{\partial}{\partial t}\left|\tilde{P}_{\mu}\right\rangle=-\left(\gamma_{\perp}+i \delta\right)\left|\tilde{P}_{\mu}\right\rangle+i \frac{|\theta|^{2}}{\hbar} N_{\mu} \hat{\mathcal{T}}_{\mu}|E\rangle .
$$

Dipole moment densities and electric field are now expanded using the same basis functions $\{|+\rangle,|-\rangle\}$. We make the following projection ansatz for each of them:

$$
\begin{gathered}
\left|\tilde{P}_{\mu}\right\rangle=\sum_{k=+,-} \tilde{P}_{\mu, k}|k\rangle, \\
|E\rangle=\sum_{k=+,-} E_{k}|k\rangle .
\end{gathered}
$$

Index $k$ should not be mistaken with the wave-number. Note, that coefficients of superpositions $\tilde{P}_{\mu, k}=\tilde{P}_{\mu, k}(t)$ and $E_{k}=E_{k}(t)$ depend only on time. Inserting the ansatz to Eq. (31) and acting using $\langle j|$ from the left, we obtain:

$$
\begin{aligned}
\frac{\partial}{\partial t} \sum_{k}\langle j \mid k\rangle \tilde{P}_{\mu, k}= & -\left(\gamma_{\perp}+i \delta\right) \sum_{k}\langle j \mid k\rangle \tilde{P}_{\mu, k} \\
& +i \frac{|\theta|^{2}}{\hbar} \sum_{k}\left\langle j\left|N_{\mu} \hat{\mathcal{T}}_{\mu}\right| k\right\rangle E_{k},
\end{aligned}
$$

Note, that acting using $\langle j|$ is mathematically equivalent to constructing the variational formulation, similarly to the framework of finite element methods.

It is useful to introduce the following time-dependent quantities:

$$
\mathcal{N}_{\mu}^{j k}=\left\langle j\left|N_{\mu} \hat{\mathcal{T}}_{\mu}\right| k\right\rangle .
$$

Mathematically, it represents the overlap integral of the spin carrier concentration. Moreover, such definition respects the polarization dependent optical response of the laser transitions. Thus, it is a natural generalization of population overlap integrals $\mathcal{N}^{j k}=\langle j|N| k\rangle$, used in Refs. [44, 47]. Overlap integrals $\mathcal{N}_{\mu}^{j k}$ defined in such a way are very important for devices, in which the optical properties of QWs depend on position. For example the strain field does not have to be uniform everywhere, resulting in different values of gain anisotropy $\mathcal{D}$ across laser. Note, that only two of them $\left(\mathcal{N}_{\uparrow}^{++}\right.$and $\left.\mathcal{N}_{\downarrow}^{--}\right)$ would be non-zero only in the absence of any linear gain anisotropy $(\mathcal{D} \rightarrow 0)$. 
Since class-C lasers are not considered in the present work, we assume that the dipole moment density follows the electric field adiabatically by taking $\partial_{t} \tilde{P}_{\mu, k}=0$. Additionally, it can be shown, that the total laser dipole moment density can be decomposed in the circular basis as:

$$
|\tilde{P}\rangle=\sum_{k}(\underbrace{\tilde{P}_{\uparrow, k}+\tilde{P}_{\downarrow, k}}_{\tilde{P}_{k}})|k\rangle .
$$

Consequently, Eq. (33) leads to:

$$
\tilde{P}_{j}=i \frac{1}{\gamma_{\perp}+i \delta} \frac{|\theta|^{2}}{\hbar} \sum_{\mu=\uparrow, \downarrow} \sum_{k=+,-} \mathcal{I}_{j}^{-1} \mathcal{N}_{\mu}^{j k} E_{k},
$$

which is the adiabatic reformulation of Eq. (9) in the given basis and where we define $\mathcal{I}_{j}=\langle j \mid j\rangle$. The interpretation of Eq. (36) is the following: generally, in the presence of a linear gain anisotropy inside the active layers, circularly-polarized fields are coupled to each other. One particular circularly-polarized field component induces not only its own dipole moment density, but also a small amount of the orthogonal one due to off-diagonal overlaps $\mathcal{N}_{\mu}^{j k}$.

\section{Photons}

The derivation of the equations of motion describing the time evolution of electric field within the spin-VCSEL cavity is based on the Eq. (11), to which the slowlyvarying envelope approximation is applied in order to get rid of second-order time derivatives. The same strategy as in the case of dipole moment density is used for the derivation of field coupled-mode equations. Namely, we express the wave equation in the bra-ket notation and then we project onto the chosen basis. Thus, we have:

$$
\begin{aligned}
\frac{\partial}{\partial t}|E\rangle= & -i \frac{\omega}{2 \varepsilon_{Q W}}|\tilde{P}\rangle \\
& -\left(\kappa+i \frac{\omega}{2}+i \frac{c^{2}}{2 \omega} \hat{\varepsilon}_{m}^{-1} \frac{\partial^{2}}{\partial z^{2}}\right)|E\rangle,
\end{aligned}
$$

where it is assumed that the active media background is described by its permittivity $\varepsilon_{Q W}$. Otherwise, the remaining part of the cavity is described generally by its permittivity tensor $\hat{\varepsilon}_{m}$, in order to describe selfconsistently any anisotropies. It is useful to define the so-called anisotropy operator $\hat{\gamma}$ :

$$
\hat{\gamma}=i \frac{\omega}{2}+i \frac{c^{2}}{2 \omega} \hat{\varepsilon}_{m}^{-1} \frac{\partial^{2}}{\partial z^{2}} .
$$

As it will be shown later, the well-known anisotropy rates used in spin-flip model, such as $\gamma_{p}$ and $\gamma_{a}$, can be calculated from normalized off-diagonal matrix elements of $\hat{\gamma}$. Moreover, we assume, that any losses in the optical cavity can be described by the decay rate $\kappa=\tilde{\kappa} / 2$, which is well-justified in the Appendix B.
Using Eq. (32) and acting on the wave equation from the left, using $\langle j|$, one thus obtains:

$$
\begin{aligned}
\frac{\partial}{\partial t} \sum_{k}\langle j \mid k\rangle E_{k}= & -i \frac{\omega}{2 \varepsilon_{Q W}} \sum_{k}\langle j \mid k\rangle \tilde{P}_{k} \\
& -\kappa \sum_{k}\langle j \mid k\rangle E_{k} \\
& -\sum_{k}\langle j|\hat{\gamma}| k\rangle E_{k} .
\end{aligned}
$$

Due to the orthogonality of circular basis, we can write $\langle j \mid k\rangle=\delta_{j k} \mathcal{I}_{k}$. Next, we use the notation $\gamma_{j k}=\langle j|\hat{\gamma}| k\rangle$ for anisotropy operator $\hat{\gamma}$ matrix elements. Using the expression for $\tilde{P}_{j}$ from Eq. (36), we derive:

$$
\begin{aligned}
\frac{\partial}{\partial t} \mathcal{I}_{j} E_{j}= & \frac{|\theta|^{2} \omega}{2 \hbar \varepsilon_{Q W}\left(\gamma_{\perp}+i \delta\right)} \sum_{\mu} \sum_{k} \mathcal{N}_{\mu}^{j k} E_{k} \\
& -\kappa \mathcal{I}_{j} E_{j}-\sum_{k} \gamma_{j k} E_{k} .
\end{aligned}
$$

Let's divide the entire equation by $\mathcal{I}_{j}$ and introduce the gain coefficient $\mathcal{G}$ defined as $\mathcal{G}(\omega)=\frac{|\theta|^{2} \omega}{2 \hbar \varepsilon_{Q W}\left(1+\alpha^{2}\right) \gamma_{\perp}}$, where $\alpha=\delta / \gamma_{\perp}$ stands for the line-width enhancement factor [56]. Finally, the general rate equation for the field amplitude $E_{j}$ is:

$$
\begin{aligned}
\frac{\partial}{\partial t} E_{j}= & \mathcal{G}(\omega)(1-i \alpha) \sum_{\mu=\uparrow, \downarrow} \sum_{k=+,-} \mathcal{I}_{j}^{-1} \mathcal{N}_{\mu}^{j k} E_{k} \\
& -\kappa E_{j}-\sum_{k=+,-} \mathcal{I}_{j}^{-1} \gamma_{j k} E_{k} .
\end{aligned}
$$

Note, that involves self-consistently the anisotropic character of the structure, not only of the passive cavity background but also of the gain media itself. Moreover, it is sensitive to any small changes in the geometry of spinVCSEL structures.

\section{Spin carriers}

We are now going to turn on to the dynamics of the spin-polarized carriers coupled to the electromagnetic field. We derive the rate equations ( 8 in total) for the overlaps $\mathcal{N}_{\mu}^{j k}$, first by only considering the coherent part of the Eq. (10), describing the electric dipole interactions. The remaining terms, such as pump rate or spin carriers decay and mixing, will be added in the heuristic manner at the end of derivation.

The interaction part of Eq. (10) may be expressed using the bra-ket notation as:

$$
\begin{aligned}
\frac{\partial}{\partial t} N_{\mu}=\ldots & +\frac{2 i}{\hbar}\left\langle E\left|\delta\left(z^{\prime}-z\right)\right| \tilde{P}_{\mu}\right\rangle \\
& -\frac{2 i}{\hbar}\left\langle\tilde{P}_{\mu}\left|\delta\left(z^{\prime}-z\right)\right| E\right\rangle,
\end{aligned}
$$

where we use the filtration property of the Dirac delta function $\delta\left(z^{\prime}-z\right)$. In order to transform $N_{\mu}$ into $\mathcal{N}_{\mu}^{j k}$, we 
act by using $\left\langle j\left|(\ldots) \hat{\mathcal{T}}_{\mu}\right| k\right\rangle$ on the both sides of Eq. (42), in which we use Eq. (32). One obtains:

$$
\begin{aligned}
\frac{\partial}{\partial t} \mathcal{N}_{\mu}^{j k}=\ldots & +\frac{2 i}{\hbar} \sum_{k} \sum_{l} \mathcal{W}_{\mu}^{j k l m} E_{l}^{*} \tilde{P}_{\mu, m} \\
& -\frac{2 i}{\hbar} \sum_{k} \sum_{l} \mathcal{W}_{\mu}^{j k l m} \tilde{P}_{\mu, l}^{*} E_{m},
\end{aligned}
$$

where the general coupling coefficients $\mathcal{W}_{\mu}^{j k l m}$ has been identified as the spatial overlap of four basis functions over the active region according to:

$$
\begin{aligned}
\mathcal{W}_{\mu}^{j k l m} & =\left\langle j\left|\left\langle l\left|\delta\left(z^{\prime}-z\right)\right| m\right\rangle \hat{\mathcal{T}}_{\mu}\right| k\right\rangle \\
& =\int_{a}\left(\boldsymbol{\varphi}_{j}^{\dagger} \hat{\mathcal{T}}_{\mu} \boldsymbol{\varphi}_{k}\right)\left(\boldsymbol{\varphi}_{l}^{\dagger} \cdot \boldsymbol{\varphi}_{m}\right) \mathrm{d} z
\end{aligned}
$$

They describe, how the generalized optical intensities $E_{k}^{*} E_{l}$ interact with spin carrier overlaps, respecting both spatial distribution of modes as well as the polarizationdependent gain. Note, that only $\mathcal{W}_{\mu}^{j k l l}$ coefficients are non-vanishing due to orthogonality of the given basis.

After having inserted $\tilde{P}_{\mu, l}$, which has been adiabatically eliminated in Eq. (33), one obtains:

$$
\begin{aligned}
\frac{\partial}{\partial t} \mathcal{N}_{\mu}^{j k}=\ldots & -\frac{4|\theta|^{2}}{\hbar^{2}\left(1+\alpha^{2}\right) \gamma_{\perp}} \sum_{l} \sum_{m} \mathcal{W}_{\mu}^{j k l l} \mathcal{I}_{l}^{-1} \\
& \times \operatorname{Re}\left\{(1-i \alpha) E_{l}^{*} E_{m} \mathcal{N}_{\mu}^{l m}\right\} .
\end{aligned}
$$

Complete rate equations for $\mathcal{N}_{\mu}^{j k}$, including pump rate overlaps defined as $\Lambda_{\mu}^{j k}=\left\langle j\left|\Lambda_{\mu} \hat{\mathcal{T}}_{\mu}\right| k\right\rangle$, dipole interaction coupling coefficient $\mathcal{K}=\frac{4|\theta|^{2}}{\hbar^{2}\left(1+\alpha^{2}\right) \gamma_{\perp}}$ and all decay and mixing rates, are:

$$
\begin{aligned}
\frac{\partial}{\partial t} \mathcal{N}_{\mu}^{j k}= & \Lambda_{\mu}^{j k}-\gamma_{\|} \mathcal{N}_{\mu}^{j k}-\gamma_{J}\left(\mathcal{N}_{\mu}^{j k}-\mathcal{N}_{\mu^{\prime}}^{j^{\prime} k^{\prime}}\right) \\
& -\mathcal{K} \sum_{l=+,-m=+,-} \sum_{\mu} \mathcal{W}_{\mu}^{j k l l} \mathcal{I}_{l}^{-1} \\
& \times \operatorname{Re}\left\{(1-i \alpha) E_{l}^{*} E_{m} \mathcal{N}_{\mu}^{l m}\right\} .
\end{aligned}
$$

Eqs. (41) and (46) form the general framework for our time-dependent modeling of spin-VCSEL structures of any spatial variation of cavity parameters and optical properties such as local anisotropies. They consist of 2 equations of motion for the field amplitudes $E_{j}$ corresponding to the two different modes, and 8 differential equations for spin carrier spatial overlaps $\mathcal{N}_{\mu}^{j k}$. Thus, they are devoted mainly for robust numerical studies of spin-lasers.

The reduced alternative of such set of equations, valid for structures properly designed, will be discussed in the following parts. Such simplification reduces not only computational cost but moreover allows to perform analytical calculations.

\section{Theory of anisotropy rates}

The important advantage of our couple mode theory is, that the entire optical cavity is treated in an unified way and all perturbations, such as anisotropies, are introduced by the overlap integrals. Equivalently, one can say, that anisotropy rates can be calculated using the matrix elements of the anisotropy operator $\hat{\gamma}$, which is derived from our Maxwell-Bloch equations. Very similar approach can be found in Ref. [57], dealing with the theory of Zeeman laser. Let's now express the general matrix element $\gamma_{j k}$ in the familiar integral notation according to:

$$
\begin{aligned}
\gamma_{j k} & =\langle j|\hat{\gamma}| k\rangle \\
& =\int_{C} \boldsymbol{\varphi}_{j}^{\dagger}\left(i \frac{\omega}{2}+i \frac{c^{2}}{2 \omega} \hat{\varepsilon}_{m}^{-1} \frac{\partial^{2}}{\partial z^{2}}\right) \boldsymbol{\varphi}_{k} \mathrm{~d} z,
\end{aligned}
$$

where the integration is performed over entire cavity. One can observe, that the different behavior can be expected from the diagonal and off-diagonal anisotropy coupling constants, because the first term under the integral will disappear, when $j \neq k$. It can be shown, that diagonal matrix elements $\gamma_{j j}$ lead to an equal frequency shifts of both laser modes in the same direction on the frequency axis. This statement applies for structures with permittivity tensors containing 'usual' anisotropies such as linear birefringence. From now on, we will not consider the diagonal contributions and we omit the first term of $\hat{\gamma}$.

In practice, the terms $\tilde{\gamma}_{j k}=\mathcal{I}_{j}^{-1} \gamma_{j k}$ have the physical meaning of anisotropy rates. We are allowed to separate the basis functions into polarization and scalar (spatial) components as $\boldsymbol{\varphi}_{j}=\boldsymbol{e}_{j} \varphi_{j}$. Using Eq. (47), one gets

$$
\tilde{\gamma}_{j k}=i \frac{c^{2}}{2 \omega} \sum_{n} \frac{\int_{n} \varphi_{j}^{*}\left(\partial_{z z}^{2} \varphi_{k}\right) \mathrm{d} z}{\int_{C}\left|\varphi_{j}\right|^{2} \mathrm{~d} z}\left[\boldsymbol{e}_{j}^{\dagger}\left(\hat{\varepsilon}_{n}^{-1}\right) \boldsymbol{e}_{k}\right],
$$

where the integration over the entire cavity has been replaced by the summation of contributions of anisotropic layers indexed by $n$. Contributions of isotropic layers are equal to zero, as can be expected. The permittivity tensor in the $n$-th layer is noted as $\hat{\varepsilon}_{n}$. Expression obtained for $\tilde{\gamma}_{j k}$ can be further simplified by describing the shape of $\varphi_{k}$ in the $n$-th layer by the superposition of respective forward and backward propagating planar waves of respective amplitudes $F_{k, n}$ and $B_{k, n}$ : $\varphi_{k}(z)=F_{k, n} \mathrm{e}^{-i q_{n}\left(z-z_{n}\right)}+B_{k, n} \mathrm{e}^{i q_{n}\left(z-z_{n}\right)}$, where $z_{n}$ is the position of $(n-1 / n)$ interface. The wave-number $q_{n}$ can be written as $q_{n}^{2}=\bar{\varepsilon}_{n}(\omega / c)^{2}$, where $\bar{\varepsilon}_{n}$ is the isotropic part of permittivity tensor. Finally, the expression for $\tilde{\gamma}_{j k}$ reads:

$$
\tilde{\gamma}_{j k}=-i \frac{\omega}{2} \sum_{n} \Gamma_{n}\left[\boldsymbol{e}_{j}^{\dagger}\left(\hat{\varepsilon}_{n}^{-1}\right) \boldsymbol{e}_{k}\right] \bar{\varepsilon}_{n},
$$

where $\Gamma_{n}$ is the optical confinement factor of the $n$-th anisotropic layer. The minus sign originates here from 
the second-order derivative of $\varphi_{k}$. As we will show later, this expression contains the well-known birefringence and dichroism rates from conventional spin-flip model. Moreover, the approach allows to derive anisotropy rates for arbitrary anisotropy, especially in structures containing more exotic anisotropies. Some of the examples can be devices with optical metasurfaces or devices with not collinear axes of anisotropies.

\section{EXTENDED SPIN-FLIP MODEL}

We will now describe in details the connection between our theoretical perturbative framework to the SFM and show that our method may generalize the previous simplified approach.

\section{A. Well-designed structures}

\section{Simplifying assumptions}

In most cases, active layers are generally located in the anti-nodes of the oscillating electromagnetic field standing-wave. Additionally, we may assume, that each active layer within the cavity, such as QW, is homogeneously excited by the same polarized field, and the polarization state of optical field does not change significantly from point to point between active layers. In this case, it does not raise any difficulty to guess approximate function which describes the spatial variation of spin carriers inside QW laser, assuming that radiative recombination takes place only in the volume of QW.

For that reason, let's write the spin-carrier populations as:

$$
N_{\mu}(z, t)=\mathcal{N}_{\mu}(t) \phi(z),
$$

where $\phi$ is the typical function one considers to describe spatial distribution of spin carriers and $\mathcal{N}_{\mu}$ is the timedependent spin carrier concentration. We choose to use a simple rectangular function. This may appear as a very strong approximation but well-justified by the fact, that the electronic wave-functions inside QW are strongly localized. The function $\phi$ has the following properties:

$$
\begin{aligned}
& \phi(z)=1 ; z \in\{a\}, \\
& \phi(z)=0 ; z \in\{C\} \backslash\{a\},
\end{aligned}
$$

where $\{a\}$ and $\{C\}$ are the sets of all points inside active layer and entire optical cavity, respectively, in this 1D approximation. Assuming such simple spatial distribution of spin carriers, their overlaps become $\mathcal{N}_{\mu}^{j k}=\mathcal{T}_{\mu}^{j k} \mathcal{N}_{\mu}$, where $\mathcal{T}_{\mu}^{j k}=\left\langle j\left|\hat{\mathcal{T}}_{\mu}\right| k\right\rangle$, and as it follows from the definition of $\phi$, the integration is performed over the active region. Moreover, it can be shown, that $\mathcal{I}_{j}^{-1} \mathcal{T}_{\mu}^{j k}=\Gamma g_{\mu}^{j k}$, where $\Gamma=\int_{a}\left|\varphi_{j}(z)\right|^{2} \mathrm{~d} z / \int_{C}\left|\varphi_{j}(z)\right|^{2} \mathrm{~d} z$ represents the conventional optical confinement factor, quantifying the relative amount of electric field energy confined in the active region. It is calculated using the scalar part of basis function $\varphi_{j}(z)$. Note, that the optical gain is polarization-dependent, which is included in the coefficient $g_{\mu}^{j k}=\boldsymbol{e}_{j}^{\dagger} \hat{\mathcal{T}}_{\mu} \boldsymbol{e}_{k}$, where $\boldsymbol{e}_{j, k}$ are the Jones vectors describing the polarization state of the basis functions. Finally, the notation $\tilde{\gamma}_{j k}=\mathcal{I}_{j}^{-1} \gamma_{j k}$ is introduced. The detailed analysis of terms $\tilde{\gamma}_{j k}$, called the anisotropy rates, will be performed in the next parts of this manuscript.

\section{Reduced rate equations}

Applying the whole above-mentioned arguments to Eqs. (41) and (46), we significantly simplify the description of spin-laser structures. Namely, the number of rate equations for spin carriers reduces to standard two, for $\mathcal{N}_{\uparrow, \downarrow}$. Resulting rate equations are:

$$
\begin{aligned}
\frac{\partial}{\partial t} E_{j}= & \mathcal{G}(\omega)(1-i \alpha) \sum_{\mu=\uparrow, \downarrow} \sum_{k=+,-} \Gamma \mathcal{N}_{\mu} g_{\mu}^{j k} E_{k} \\
& -\kappa E_{j}-\sum_{k=+,-} \tilde{\gamma}_{j k} E_{k} \\
\frac{\partial}{\partial t} \mathcal{N}_{\mu}= & -\gamma_{\|}\left(\mathcal{N}_{\mu}-\mathcal{N}_{0 \mu}\right)-\gamma_{J}\left(\mathcal{N}_{\mu}-\mathcal{N}_{\mu^{\prime}}\right) \\
& -\mathcal{K} \sum_{l=+,-} \sum_{m=+,-} \Gamma \operatorname{Re}\left\{g_{\mu}^{l m} E_{l}^{*} E_{m}\right\} \mathcal{N}_{\mu}
\end{aligned}
$$

where $\mathcal{N}_{0 \mu}$ stands for unsaturated spin carrier concentration at $\mu$-th spin channel. The two additional assumptions has been used. First, it was assumed, that the basis functions are normalized in such way, that they satisfy $\boldsymbol{\varphi}_{j}^{\dagger} \cdot \boldsymbol{\varphi}_{j} \cong 1$ inside the active layers, which are very thin compared to the typical wavelength $(\lambda \cong 1 \mu \mathrm{m})$. Second, the original set of coupled-mode equations has been constructed in the way, that $\mathcal{T}_{\mu}^{j k}=\mathcal{T}_{\mu^{\prime}}^{j^{\prime} k^{\prime}}$ must hold (for example $\mathcal{T}_{\uparrow}^{++}=\mathcal{T}_{\downarrow}^{--}$).

\section{B. Spin-flip model with linear gain anisotropy}

The important consequence of Eqs. (52) and (53) is the possibility to derive the extension of the well-known SFM to some more general situations.

i) We extend the SFM to include the gain anisotropy different than circular gain dichrosim (due to spin carrier imbalance), such as the linear gain dichroism due to strain inside QW.

ii) The second extension originates from the fact, that the cavity-related quantities such as anisotropy rates can be calculated self-consistently. This provides a clear rigorous background to SFM.

iii) Moreover, the extended SFM can be straightforwardly generalized to respects the shape of the optical mode within the cavity, especially in active region. 
In order to derive the extended SFM, the field and population variables must be re-scaled in an appropriate way. To do so, we introduce the populations $N, m$ and mode amplitudes $A_{ \pm}$, in the similar manner as San Miguel et al. [30], defined as:

$$
\begin{aligned}
\mathcal{N}_{\uparrow, \downarrow} & =\frac{\kappa}{\Gamma \mathcal{G}}(N \pm m), \\
E_{ \pm} & =\sqrt{\frac{2 \gamma_{\|}}{\mathcal{K} \Gamma}} A_{ \pm} \exp (-i \alpha \kappa t) .
\end{aligned}
$$

We get rid of fundamental constants and coupling coefficients, keeping just the main structure of rate equations necessary for description of important laser features.

Before we find the rate equations for $A_{ \pm}, N, m$, it is useful to study the polarization-dependent coefficients $g_{\mu}^{j k}$. Due to the specific basis we have chosen, and relatively small values of gain anisotropy parameter $\mathcal{D}$ (up to $\mathcal{D}=0.1$, we are allowed to neglect two of them, namely $g_{\uparrow}^{--}$and $g_{\downarrow}^{++}$. From the definition, it can be shown, that they are equal to each other: $\boldsymbol{e}_{-}^{\dagger} \hat{\mathcal{T}}_{\uparrow} \boldsymbol{e}_{-}=\boldsymbol{e}_{+}^{\dagger} \hat{\mathcal{T}}_{\downarrow} \boldsymbol{e}_{+}=$ $\mathcal{D}^{2} /\left(1+\mathcal{D}^{2}\right)$. The remaining coefficients $g_{\mu}^{j k}$ take only two different values and according to calculations, we define: $g=1 /\left(1+\mathcal{D}^{2}\right)$ and $\tilde{g}=2 \mathcal{D} /\left(1+\mathcal{D}^{2}\right)$. Moreover, we neglect the anisotropy rates $\tilde{\gamma}_{ \pm \pm}$, which induce the frequency shift of two modes in the same direction, but not splitting between them.

Thus, the relations from Eq. (54) are applied to Eqs. (52) and (53), from which one obtains, after performing the simple algebraic operations, the so-called extended spin-flip model:

$$
\begin{gathered}
\dot{A}_{+}=\kappa(1-i \alpha)\left\{[g(N+m)-1] A_{+}+\tilde{g} N A_{-}\right\}-\tilde{\gamma}_{+-} A_{-}, \\
\dot{A}_{-}=\kappa(1-i \alpha)\left\{[g(N-m)-1] A_{-}+\tilde{g} N A_{+}\right\}-\tilde{\gamma}_{-+} A_{+}, \\
\dot{N}=\gamma_{\|}\left(N_{0}-N\right)-\gamma_{\|}\left\{\left[g\left(I_{+}+I_{-}\right)+\tilde{g} I_{ \pm}\right] N+g\left(I_{+}-I_{-}\right) m\right\}, \\
\dot{m}=\gamma_{\|} \mathcal{P}_{J} N_{0}-\left(\gamma_{\|}+2 \gamma_{J}\right) m-\gamma_{\|}\left\{\left[g\left(I_{+}+I_{-}\right)+\tilde{g} I_{ \pm}\right] m+g\left(I_{+}-I_{-}\right) N\right\},
\end{gathered}
$$

where the optical intensities $I_{+}=\left|A_{+}\right|^{2}, I_{-}=\left|A_{-}\right|^{2}$ and $I_{ \pm}=2 \operatorname{Re}\left\{A_{+}^{*} A_{-}\right\}$have been defined. The overall pumping rate is described by unsaturated carrier concentration $N_{0}$. The fact that the linewidth enhancement factor $\alpha$ may be polarization-dependent has been neglected here.

Note, that in the absence of any linear gain anisotropy $(\mathcal{D}=0)$, the coefficients $g$ and $\tilde{g}$ reduce to $g=1$ and $\tilde{g}=0$, which gives the conventional SFM with generalized anisotropy rates $\tilde{\gamma}_{ \pm \mp}$, which consist of the birefringence rate $\gamma_{p}$ and the dichroism rate $\gamma_{a}$ as a special case, as it will be shown.

One of the predictions of the extended SFM is an additional contribution to total frequency splitting between linear lasing modes. It is due to phase-amplitude coupling in the presence of the linear gain anisotropy $\mathcal{D}$. Standard derivation leads to the approximate expression:

$$
\delta \omega \cong 2 \alpha \kappa \mathcal{D}
$$

valid for small values of $\mathcal{D}$, which is formally equivalent to a general expression given in literature for such kind of anisotropy [58].

One can expect new effects arising due to $\mathcal{D} \neq 0$. Detailed qualitative analysis of extended SFM, such as bifurcation analysis or calculation of modulation response, is out of the scope of the present paper. Nevertheless, gain anisotropy should strongly influence beating fre- quency between circular modes.

It is important to note, that if we used a different basis to derive the extended SMF, we would obtain a different set of differential equations. However, the physics, which is modeled, would be the same as long as we neglect terms in the second order of gain anisotropy $\mathcal{D}$.

\section{Linear birefringence and dichroism rates}

The mathematical framework, developed in this paper, allows to calculate realistic values of anisotropy rates such as birefringence rate $\gamma_{p}$ and dichroism rate $\gamma_{a}$. They can be calculated not only using the rigorous matrix formalism [19], that has been re-formulated within this paper, but more importantly in a self-consistent way within the framework of the time-dependent coupled mode theory. This subsection is based on the computational rules, introduced in the Sec. IV C. We will derive approximate analytic expressions for $\gamma_{p}$ and $\gamma_{a}$, which may provide useful physical insights.

In order to derive and validate this approach, we consider the single-QW structure, as depicted in Fig. 5. To simplify, this structure contains only a single anisotropic layer (apart from the possible anisotropies in the active layer), which will be placed in two different locations within the optical cavity. This is emphasized by respec- 
tive red and blue layers with reduced coordinates $\zeta=0.1$ and $\zeta=0.15$ in the local reference frame, as shown in the inset. Let's write down the permittivity tensor of such layer without $z$-components, since the off-axis wave propagation along $z$ is neglected here. Note, that the permittivity tensor is expressed in the $\{[110],[1 \overline{1} 0]\}$ basis according to:

$$
\hat{\varepsilon}_{A}=\left[\begin{array}{cc}
\varepsilon_{A, x x} & 0 \\
0 & \varepsilon_{A, y y}
\end{array}\right] .
$$

Next, we consider a particular linear birefringence and linear dichroism inside a given layer. Material birefringence and dichroism are quantified using parameters $\delta \varepsilon_{r}$ and $\delta \varepsilon_{i}$, respectively: $\delta \varepsilon_{r}=\operatorname{Re}\left\{\varepsilon_{A, x x}-\varepsilon_{A, y y}\right\}$ and $\delta \varepsilon_{i}=$ $\operatorname{Im}\left\{\varepsilon_{A, x x}-\varepsilon_{A, y y}\right\}$. Both parameters are positive real numbers by the convention. We calculate the off-diagonal normalized matrix element $\tilde{\gamma}_{+-}$of the anisotropy operator $\hat{\gamma}$. Using the Eq. (49), one obtains:

$$
\tilde{\gamma}_{+-}=-i \frac{\omega}{2} \Gamma_{A}\left[e_{+}^{\dagger}\left(\hat{\varepsilon}_{A}^{-1}\right) e_{-}\right] \bar{\varepsilon}_{A},
$$

where $\Gamma_{A}$ stands for the optical confinement factor of anisotropic layer and $\bar{\varepsilon}_{A}$ is the absolute value of isotropic part of permittivity tensor. Straightforward calculation, in which the convention $\boldsymbol{e}_{ \pm}=[1, \mp i]^{T} / \sqrt{2}$ is used, leads to:

$$
\tilde{\gamma}_{+-}=\Gamma_{A} \frac{\omega}{2}\left(\frac{\delta \varepsilon_{i}+i \delta \varepsilon_{r}}{2 \varepsilon_{A, x x} \varepsilon_{A, y y}}\right) \bar{\varepsilon}_{A}
$$

In order to simplify this expression for $\tilde{\gamma}_{+-}$, we use the following approximation $\varepsilon_{A, x x} \varepsilon_{A, y y} \cong \bar{\varepsilon}_{A}^{2}$, which is valid in the case of $\delta \varepsilon_{r, i}<<\bar{\varepsilon}_{A}$. Within this approximation, one can see, that the expression we have derived consists of real and imaginary part contributions. We will show numerically, that this can be interpreted in the following way: $\operatorname{Re}\left\{\tilde{\gamma}_{+-}\right\}=\gamma_{a}$ and $\operatorname{Im}\left\{\tilde{\gamma}_{+-}\right\}=\gamma_{p}$. Namely, that the real and imaginary parts of $\tilde{\gamma}_{+-}$are equal to the dichroism rate $\gamma_{a}$ and the birefringence rate $\gamma_{p}$, respectively. They are given approximately by the simple relations:

$$
\begin{aligned}
& \gamma_{a} \cong \Gamma_{A}\left(\frac{\omega}{2}\right) \frac{\delta \varepsilon_{i}}{2 \bar{\varepsilon}_{A}}, \\
& \gamma_{p} \cong \Gamma_{A}\left(\frac{\omega}{2}\right) \frac{\delta \varepsilon_{r}}{2 \bar{\varepsilon}_{A}} .
\end{aligned}
$$

Such formulas are rather intuitive concerning polarization anisotropies in VCSELs, but also agree qualitatively with alternative expressions derived in the past, to some extent [25, 38]. However, according to our knowledge, they have never been derived explicitly in this form.

\section{Numerical validation}

In this section, we compare the predictions of analytic expressions directly with precise matrix formalism (see

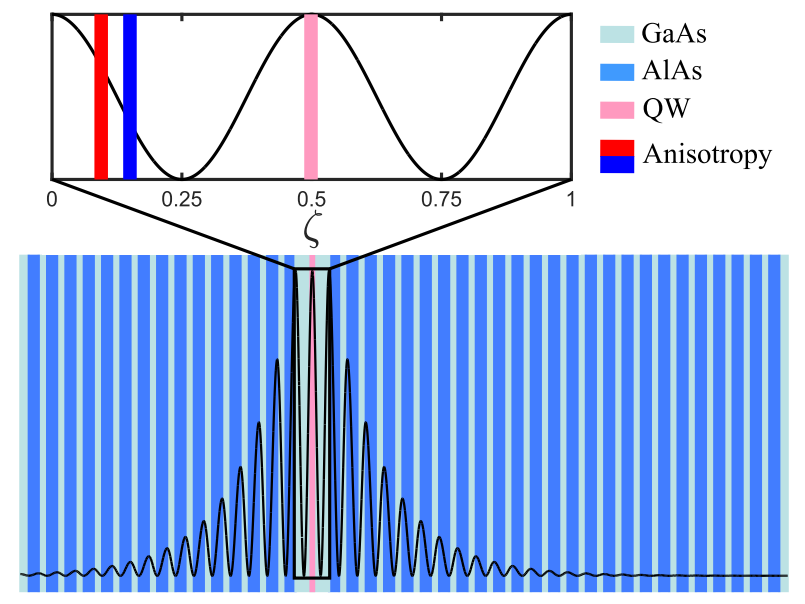

FIG. 5. Scheme of a simple single-QW structure, used for numerical validation, with the electric field distribution calculated using the modified matrix formalism. The detail shows the position of active layer and two possible positions [red $(\zeta=0.1)$, blue $(\zeta=0.15)$ ] of anisotropic passive layer within the $\lambda$-cavity field. The local reference frame is described by $\zeta$.

Sec. III), showing the robustness of our coupled mode theory implemented here. We will focus now on the numerical predictions of experimentally-related quantities such as the frequency splitting between orthogonal linear modes in the absence of any spin injection. Another quantity of interest is the splitting of threshold pump rates, or threshold carrier concentrations of such modes. Finally, the polarization state of emitted modes is studied.

The formalism is demonstrated on a single-QW spinVCSEL structure, as depicted in Fig. 5. It consists of a single active layer with thickness of $d_{Q W}=10 \mathrm{~nm}$, which models the QW. The short cavity is placed between two Bragg mirrors, composed of 15 and 25 units of $\mathrm{GaAs} / \mathrm{AlAs}$, respectively, and designed for wavelength of around $\lambda=1.005 \mu \mathrm{m}$. Apart from the active layer, the cavity contains also the anisotropic layer of thickness $d_{A}=25 \mathrm{~nm}$, with linear birefringence and linear dichroism described by non-zero $\delta \varepsilon_{r, i}$. Optical constants are: $\varepsilon_{G a A s}=12.25, \varepsilon_{A l A s}=8.68, \varepsilon_{Q W}=12.96$ and $\bar{\varepsilon}_{A}=\varepsilon_{G a A s}[59]$. We will show, how some of main consequences of even the smallest change in the geometry of the device can be predicted with sufficiently high precision. It is shown in Sec. VI, how exactly the anisotropic elements inside laser cavity can be technologically important.

\section{Frequency splitting}

We now consider the given single-QW structure free of any anisotropies beside linear birefringence. It can be derived from the SFM, that the frequency splitting is 


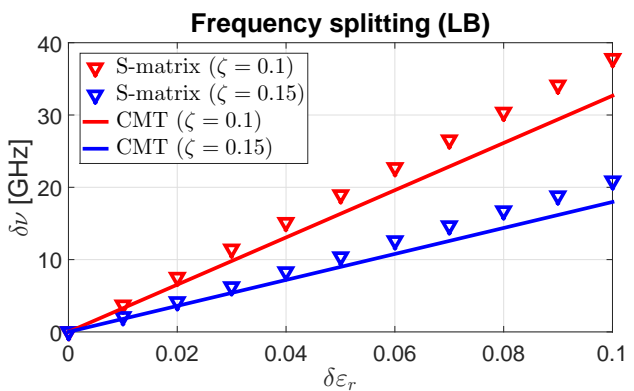

(a)

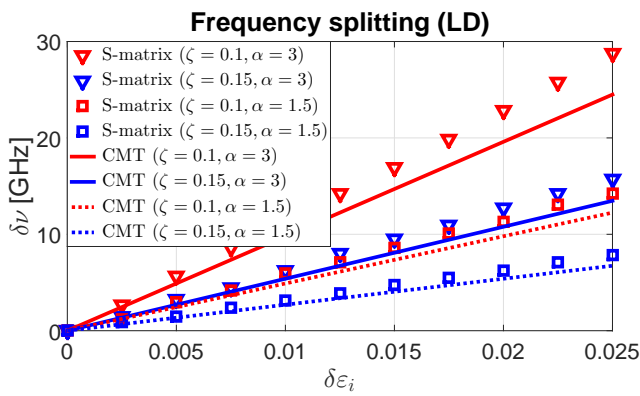

(b)
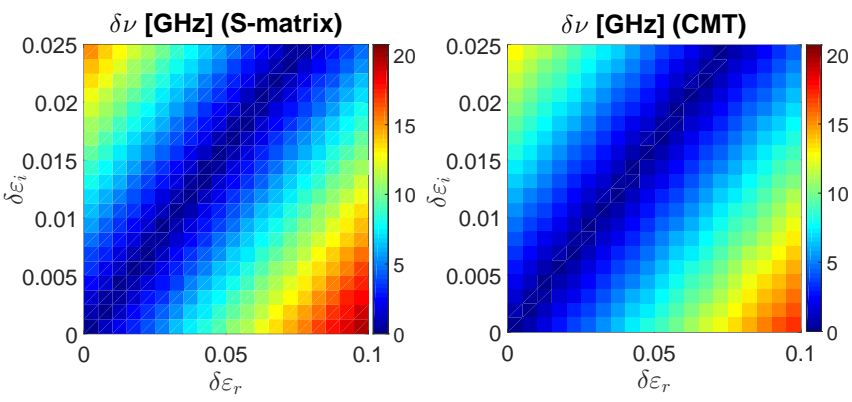

(c)

FIG. 6. The frequency splitting calculated as a function of the birefringence parameter $\delta \varepsilon_{r}$ (a), of the dichroism parameter $\delta \varepsilon_{i}$, due to non-zero Henry's factor $\alpha$ (b), and of the both anisotropy parameters (c). The results obtained using our matrix formalism are used as a reference to evaluate the precision of the method.

given as $\delta \nu=\gamma_{p} / \pi$ [31], where we calculate $\gamma_{p}$ as a function of $\delta \varepsilon_{r}$ according to approximate formula given in Eq. (63). Direct numerical comparison with calculation based on our matrix formalism is shown in Fig. 6(a). Particular colors of the lines refer to position of anisotropic layer within the optical field according to Fig. 5. The values of the confinement factor of anisotropic layer are $\Gamma_{\text {red }}=0.0269$ and $\Gamma_{\text {blue }}=0.0148$. One can observe a small discrepancy between the scattering matrix method and our coupled mode theory. This fact can be attributed to the number of approximations we have used in order to derive the analytic formulas. However, in principle, they do not have to be used while applying our recipe of coupled mode theory.

Moreover, the extended SFM can be efficiently combined with our matrix algebra to obtain some of the re-

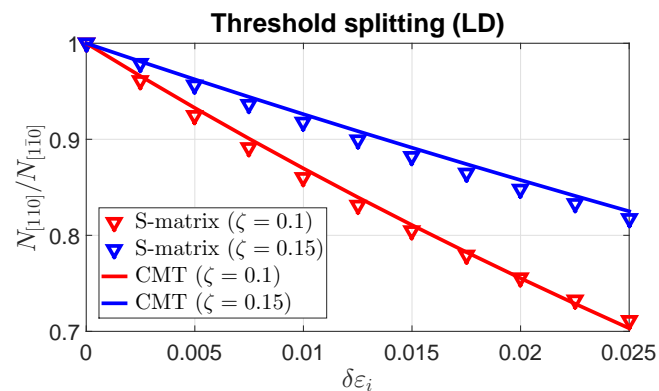

(a)

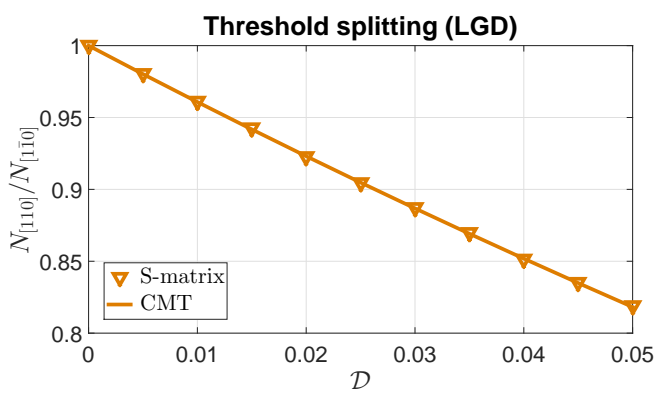

(b)

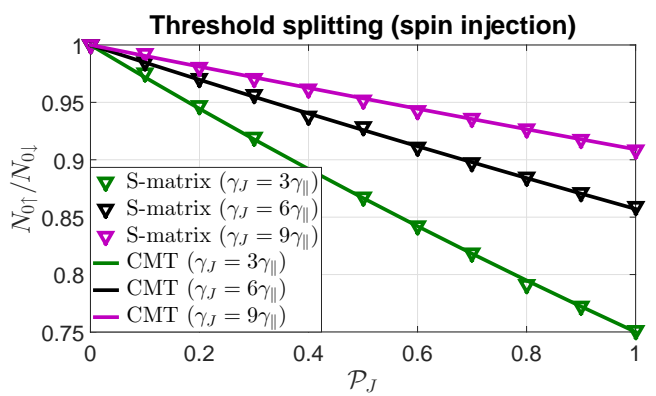

(c)

FIG. 7. (a) The calculation of ratio $N_{[110]} / N_{[1 \overline{1} 0]}$ as a function of the linear dichroism parameter $\delta \varepsilon_{i}$. (b) The calculation of ratio $N_{[110]} / N_{[1 \overline{1} 0]}$ as a function of the linear gain anisotropy $\mathcal{D}=\delta \varepsilon_{a, i} /(2 \bar{\chi})$. (c) The ratio of threshold pumping rates $N_{0 \uparrow} / N_{0 \downarrow}$ in the structure with circular gain dichroism, due to electron spin imbalance, but without additional linear anisotropies.

quired quantities with a better precision. For example, one can compute the mode thresholds and frequencies, from which it is possible to straightforwardly extract the anisotropy rates.

Another contribution to the frequency splitting originates from the phase-amplitude coupling. In this case, the SFM predicts the value $\delta \nu=(\alpha / \pi) \gamma_{a}[31]$, where the dichroism rate is the function of dichroism parameter $\delta \varepsilon_{i}$, as shown by the coupled mode theory. Note, that the birefringence in the layer is switched off: $\delta \varepsilon_{r}=0$. The results, together with the comparison to rigorous matrix method, is depicted in Fig. 6(b). Two different realistic values of Henry's factor $\alpha$ has been used, so the magnitude of phase-amplitude coupling can be controlled. Qualitatively, the results are in the perfect agreement. 
The inaccuracy seems to be affected by the same way, since the ratio of data obtained by both methods is the same as for our previous calculation.

Finally, the combined contribution of both local anisotropy parameters $\delta \varepsilon_{r, i}$ has been studied. The absolute value of frequency difference is $\delta \nu=\left|\gamma_{p}-\alpha \gamma_{a}\right| / \pi$. A very good agreement between both methods has been obtained too, as shown in Fig. 6(c). The results were calculated for $\zeta=0.15$.

It should be noted, that the calculations using the linear basis functions have been performed as well. We decided to use the option of two orthogonal linearlypolarized basis functions with degenerate frequencies. In this case, the coupled mode theory gives almost identical results to those using circular basis.

\section{Threshold splitting}

The calculation concerning the effects of anisotropies on lasing threshold is not that straightforward as in the case of frequency splitting. The validity of coupled mode theory may be determined by calculating the ratio $N_{[110]} / N_{[1 \overline{1} 0]}$, where $N_{[110],[1 \overline{1} 0]}$ are the threshold carrier concentrations for linear laser modes oscillating along [110] and [1̄o] crystallographic axes, respectively. The prediction of spin-flip model is $N_{[110]} / N_{[1 \overline{1} 0]}=$ $\left(\kappa-\gamma_{a}\right) /\left(\kappa+\gamma_{a}\right)$. Note, that in the case of matrix formalism, one has to calculate the ratio $\bar{\chi}_{[110]} / \bar{\chi}_{[1 \overline{1} 0]}$, since $\bar{\chi} \propto N_{\uparrow}+N_{\downarrow}$ or equivalently $N_{0 \uparrow}+N_{0 \downarrow}$, because we consider laser at or near threshold. The results, depicted in Fig. 7(a), show an excellent agreement.

Next, we consider the optical anisotropies located solely inside the active layers: circular gain dichroism, induced by electron spin imbalance, and linear gain dichroism. We neglect any additional passive anisotropies now. One should expect significant differences in the pumping rates at respective thresholds (or threshold carrier concentrations) of particular modes in the presence of gain anisotropies.

Fig. $7(\mathrm{~b})$ displays the calculation of $N_{[110]} / N_{[1 \overline{1} 0]}$, where $N_{[110]}=1 /(g+\tilde{g})$ and $N_{[1 \overline{1} 0]}=1 /(g-\tilde{g})$, according to extended spin-flip model. Adjustable parameter is $\mathcal{D}$. Note, that in the language of permittivities one derives $\delta \varepsilon_{a, i}=2 \bar{\chi} \mathcal{D}$, where $\delta \varepsilon_{a, i}$ is the difference of imaginary parts of permittivities along anisotropy principal axes (see Sec. III). Numerical values obtained using our extended SFM are in excellent agreement with those extracted using matrix formalism. The similar calculation has been performed for circular modes under spinpolarized pumping $\left(\mathcal{P}_{J}\right)$ for several values of spin-mixing rate $\gamma_{J}$.

\section{Polarization eigenmodes at threshold}

Next, we show, that our extended SFM gives good results even concerning polarization state of possible
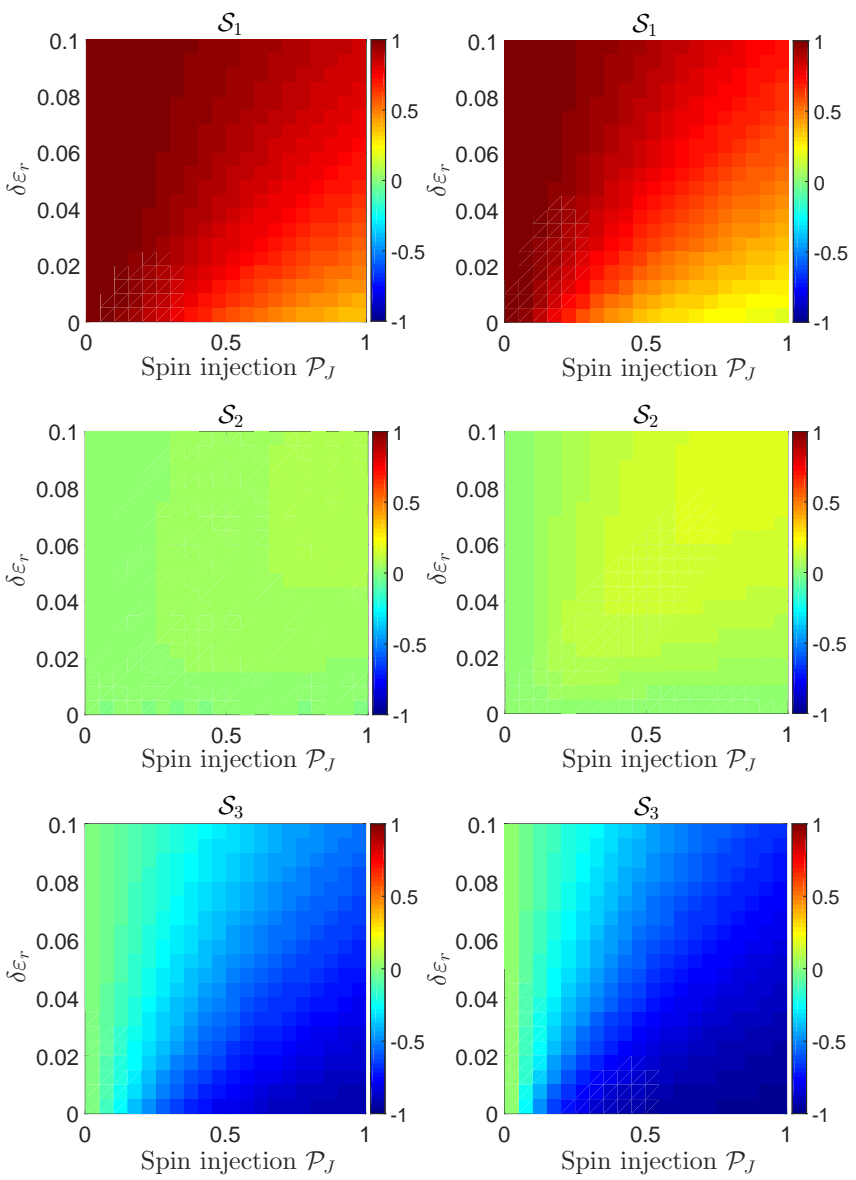

FIG. 8. Components of reduced Stokes vector $\mathcal{S}=$ $\left[\mathcal{S}_{1}, \mathcal{S}_{2}, \mathcal{S}_{3}\right]^{T}$ of single laser eigenmode calculated using rigorous matrix formalism (left column) and using the extended spin-flip model based on the coupled-mode theory (right column).

threshold modes, which may be the most general in this case. We consider the linear birefringence and linear dichroism in passive layer, linear gain anisotropy in active layer and additionally spin-polarized electron injection. Very similar calculation has been presented in our previous contribution [50], in which we have used scattering matrix formalism to extract dynamics-related quantities for conventional SFM. However, in this case, our extended SFM based on coupled mode theory, is computationally self-consistent. Thus, the results of robust matrix formalism are not required in order to obtain comparable results.

Let's transform equations for field amplitudes from extended SFM to the $\{\boldsymbol{x}, \boldsymbol{y}\}$ basis, in which the field amplitude components are $\boldsymbol{A}=\left[A_{x}, A_{y}\right]^{T}$. Resulting rate equations can be expressed compactly as:

$$
\frac{\partial}{\partial t} \boldsymbol{A}=\hat{\Omega} \boldsymbol{A}
$$

where $\hat{\Omega}$ is certain time-evolution operator. It can be shown, that the polarization eigenmodes, we are looking 
for, are the eigenvectors of operator $\hat{\Omega}$.

Analytically extracted eigenvectors are used in order to calculate components of Stokes vector $\mathcal{S}$ as functions of spin injection polarization degree $\mathcal{P}_{J}$ and birefringence parameter $\delta \varepsilon_{r}$ (and the small $\delta \varepsilon_{i}=\delta \varepsilon_{r} / 10$ ) with fixed $\mathcal{D}=0.025$ and $\gamma_{J}=3 \gamma_{\|}$, which belongs to range of values observed experimentally at low temperatures. Otherwise, values of $\gamma_{J}$ are much larger [60]. The results are shown in Fig. 8, together with rafined calculations based on the scattering matrix formalism. Compared to the results from Ref. [50], in which we have compared the matrix approach with the conventional spin-flip model, one can see, that separation of amplitude anisotropy into passive (linear dichroism) and active (linear gain anisotropy) contributions increases the precision.

\section{APPLICATION: SPIN-VCSELS FOR ULTRAFAST DATA TRANSMISSION AND NOVEL THZ SOURCES}

As previously noted, one promising way to increase the speed of optical communication is to use highlybirefringent spin-VCSELs for the polarization modulation. In this section, we apply the developed theoretical tools to design and optimize conceptual spin-VCSEL structure with 1D grating, which locally induces extremely large linear birefringence and thus, large frequency splitting between co-existing linear modes. It is an alternative option to recently demonstrated methods based on heating or bending the structures [13, 61] and implementing the surface grating $[62,63]$.

\section{A. Spin-VCSEL with intra-cavity grating}

In order to reach frequency splitting in the range of $\mathrm{THz}$, we propose to fabricate a spin-VCSEL structure with high-contrast intra-cavity grating, as shown in Fig. 9. It is based on a resonant $2 \lambda$-cavity made of GaAs, with 4 InGaAs QWs and AlGaAs/air grating. The Bragg mirrors are made of 15 and 25 units of GaAs/AlAs. The structure is optimized to emit at $\lambda=1300 \mathrm{~nm}$. Optical constants are: $\varepsilon_{G a A s}=11.56$, $\varepsilon_{\text {AlAs }}=8.41, \varepsilon_{\text {InGaAs }}=12.32$ and $\varepsilon_{\text {AlGaAs }}=10.69$ [59]. The design has been performed using the numerical recipe introduced in Sec. III.

This type of structures are usually fabricated in three steps: i) initial epitaxial growth of substrate Bragg mirror and MQW active region, ii) fabrication of grating using for example electron-beam litography and iii) epitaxial regrowth of the rest of the structure [64, 65]. Alternative method to produce such structure could be the wafer bonding.

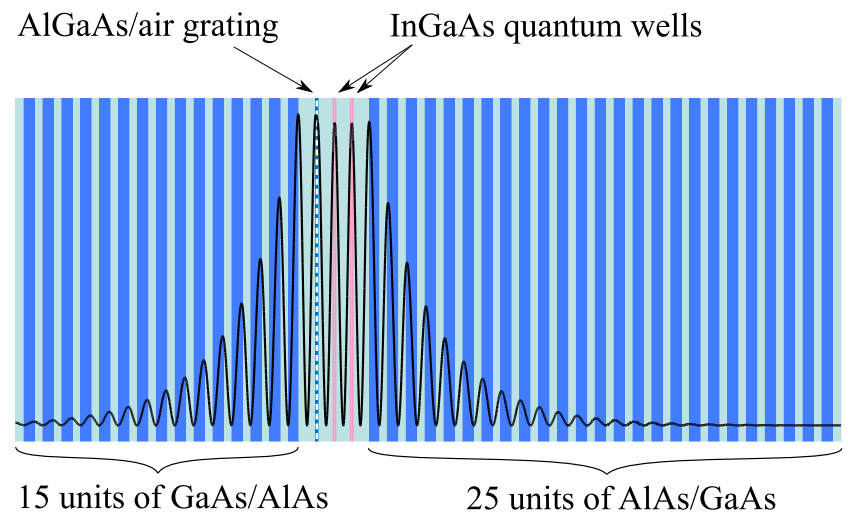

FIG. 9. A scheme of $\lambda=1300 \mathrm{~nm}$ VCSEL with 4 InGaAs QWs and intra-cavity AlGaAs/air grating.

\section{B. Ultrafast polarization modulation}

We use effective medium approximation (EMA), valid for large wavelengths [66], to describe the effective anisotropy of grating. For simplicity, we consider the grating to be the only source of anisotropy. In such case of lamelar grating, the ordinary and extraordinary permittivities $\varepsilon_{o, e}$ are given by $\varepsilon_{o}=f \varepsilon_{a}+(1-f) \varepsilon_{b}$ and $\varepsilon_{e}^{-1}=f \varepsilon_{a}^{-1}+(1-f) \varepsilon_{b}^{-1}$, where $f$ is the fill factor and $\varepsilon_{a, b}$ are bulk permittivities of two materials composing the grating [67]. In the case of the structure depicted in Fig. 9, they are $\varepsilon_{a}=\varepsilon_{A l G a A s}$ and $\varepsilon_{b}=\varepsilon_{a i r}$.

The frequency splitting induced by grating, calculated using our matrix formalism, is shown in Fig. 10(a) for variable grating thickness and fill factor. We show, that using intra-cavity grating it is possible to reach frequency splitting $\delta \nu>1 \mathrm{THz}$, if one correctly chooses the composition of the grating. Note, that such large anisotropy is beyond the validity of the coupled mode theory (or extended SFM), because of extremely large birefringence $\delta \varepsilon_{r}$ in grating, which is far beyond the perturbative regime.

However, the predictions of the presented matrix formalism allow to calculate the birefringence rate $\gamma_{p}=\pi \delta \nu$, which is used in SFM. In Fig. 10(b), we show the simulated polarization modulation described using degree of circular polarization $\mathcal{P}_{c}(t)=\left[I_{+}(t)-I_{-}(t)\right] /\left[I_{+}(t)+\right.$ $\left.I_{-}(t)\right]$. We study two different positions of grating within the cavity. The parameters used in simulations are $N_{0}=5, \mathcal{P}_{J}=0.5, \alpha=3, \gamma_{\|}=1 \mathrm{GHz} \kappa=133 \mathrm{GHz}$, $g=1, \tilde{g}=0(\mathcal{D}=0)$ and fill factor $f=0.25$ in both cases. The speed of modulation is limited by the value of spin-mixing rate $\gamma_{J}$, which was chosen to be $\gamma_{J}=500 \gamma_{\|}$ here. Nowadays, there is significant experimental effort to make spin-mixing rate as large as possible, in contrary to conventional spintronics.

Fig. 10(b) also demonstrates strong sensitivity of resulting polarization modulation speed to location of the grating layer in the multilayer structure. One can see, that even very small shift in position of grating leads to 


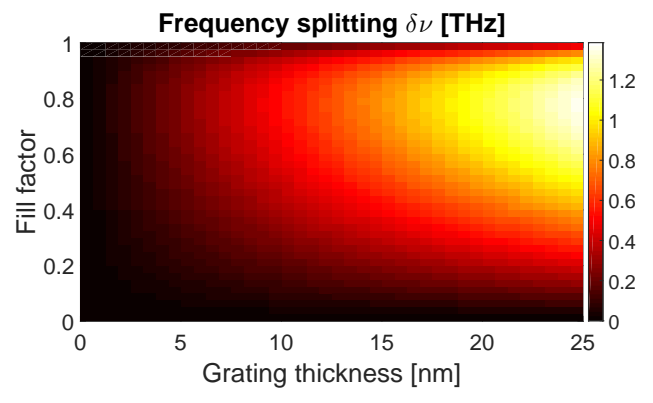

(a)

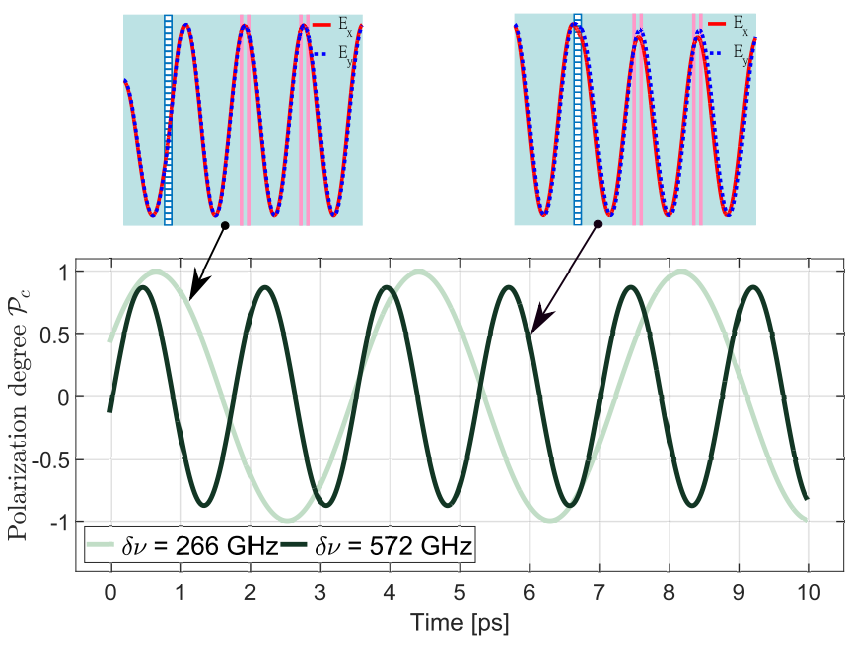

(b)

FIG. 10. (a) Calculated frequency splitting between orthogonally polarized laser modes as a function of grating thickness and fill factor of AlGaAs inside the grating. (b) Simulated polarization modulation of spin-VCSEL with grating for two different positions of grating within the cavity.

very large change of polarization degree oscillations. In near future, our theoretical framework will be extended to structures with lateral periodicity by incorporating rigorous coupled-wave analysis (RCWA), for which our approach is well-suited [68].

From the broader application perspective, the possibility of generating coherent $\mathrm{THz}$ radiation using highlybirefringent spin-VCSELs seems very promising. The existing $\mathrm{THz}$ sources are too large, or they can only operate at very low temperatures. Alternatively, they require the use of more than one external laser. All of these disadvantages could be eventually solved using highlyanisotropic spin-lasers, in which the beat-node signal in the $\mathrm{THz}$ range coming from interference of orthogonally polarized modes, could be used to generate $\mathrm{THz}$ radiation by means of photo-mixing [14]. As depicted in Fig. 10(a), we reached the frequency splitting of orthogonal linear modes close to $\delta \nu \cong 1.4 \mathrm{THz}$ for the grating fill factor $f=0.75$ for modeled spin-VCSEL with intra-cavity grating.

\section{CONCLUSION AND PERSPECTIVES}

We have formulated the semi-classical model of spinlaser with particular focus on local optical anisotropies and highly-birefringent structures. It has been used to develop:

i) the steady-state robust matrix description of spinVCSELs. This can be further generalized to describe the steady-state operation of spin-VCSELs pumped above threshold, including saturation effects. Important part will be to use more realistic description of QW gain and include lateral effects in electromagnetic wave propagation. It will allow to study the mode competition in a rigorous way. Concerning the $\mathrm{THz}$ applications, it is suitable for direct implementation of periodic structures such as birefringent gratings by means of RCWA.

ii) the coupled mode theory, which allows to treat spinVCSEL structures layer-by-layer. It is sensitive to any small changes in optical and geometric properties of laser cavity and gain media. We use the vectorial eigenmodes of active structures, extracted using matrix formalism, in contrary to previous approaches. The only input parameters are the optical and geometric properties of particular layers. Our model leads to the extended spin-flip model with polarization-dependent gain. In future, it will be worth to perform detailed analysis of effects arising from additional terms due to linear gain anisotropy.

In this paper, we have proposed and designed conceptual structure of spin-VCSEL with intra-cavity grating. Such structures have potential to be of significant importance for $\mathrm{THz}$ photonics: i) as ultrafast polarization modulators for data transfer and ii) compact tunable sources of $\mathrm{THz}$ radiation. Robust matrix formalism together with coupled mode theory have been proven to be precise tools for design and optimization of future spinVCSEL structures.

\section{Appendix A: Transfer and scattering matrix formalism}

Steady-state electromagnetic field propagation inside multilayer structures such as spin-VCSELs can be efficiently described using the Yeh's matrix approach. The basic idea is that one solves the wave equation for each layer separately and these solutions are connected by applying relevant boundary conditions. The frequency domain wave equation for the electric field amplitude $\boldsymbol{E}_{0}^{(n)}$ inside any general anisotropic media is:

$$
\mathbf{W}^{(n)} \boldsymbol{E}_{0}^{(n)}=0,
$$

where $\mathbf{W}^{(n)}=(\omega / c)^{2} \hat{\varepsilon}^{(n)}-\left[\boldsymbol{q}^{(n)}\right]^{2}+\boldsymbol{q}^{(n)} \otimes\left[\boldsymbol{q}^{(n)}\right]^{T}$ is the wave equation operator with the permittivity tensor $\hat{\varepsilon}^{(n)}$ and wave-vector $\boldsymbol{q}^{(n)}$ specific for each layer. The $z$ components of $\boldsymbol{q}^{(n)}$ are extracted from the condition for the non-trivial solution:

$$
\operatorname{det}\left[\mathbf{W}^{(n)}\right]=0 .
$$


Due to the symmetry of the considered class of media, one obtains four propagation constants $\boldsymbol{q}_{z j}^{(n)}$, where $j=$ 1,3 refer to forward propagating modes and $j=2,4$ to backward propagating modes. Solving Eq. (A1) gives particular amplitudes $\boldsymbol{E}_{0 j}^{(n)}=A_{j}^{(n)} \boldsymbol{e}_{j}^{(n)}$, where $\boldsymbol{e}_{j}^{(n)}$ is the eigen-polarization vector.

Boundary conditions at the interface between $(n-1)$ and $(n)$ layers can be expressed using matrices as:

$$
\mathbf{D}^{(n-1)} \boldsymbol{A}^{(n-1)}=\mathbf{D}^{(n)} \mathbf{P}^{(n)} \boldsymbol{A}^{(n)},
$$

where $\boldsymbol{A}^{(n)}$ is the vector of amplitudes, $\mathbf{D}^{(n)}$ and $\mathbf{P}^{(n)}$ are dynamic and propagation matrix, respectively. They are:

$$
\begin{gathered}
\mathbf{D}^{(n)}=\left[\begin{array}{cccc}
\boldsymbol{e}_{x 1}^{(n)} & \boldsymbol{e}_{x 2}^{(n)} & \boldsymbol{e}_{x 3}^{(n)} & \boldsymbol{e}_{x 4}^{(n)} \\
\boldsymbol{h}_{y 1}^{(n)} & \boldsymbol{h}_{y 2}^{(n)} & \boldsymbol{h}_{y 3}^{(n)} & \boldsymbol{h}_{y 4}^{(n)} \\
\boldsymbol{e}_{y 1}^{(n)} & \boldsymbol{e}_{y 2}^{(n)} & \boldsymbol{e}_{y 3}^{(n)} & \boldsymbol{e}_{y 4}^{(n)} \\
\boldsymbol{h}_{x 1}^{(n)} & \boldsymbol{h}_{x 2}^{(n)} & \boldsymbol{h}_{x 3}^{(n)} & \boldsymbol{h}_{x 4}^{(n)}
\end{array}\right], \\
\mathbf{P}^{(n)}=\left[\begin{array}{cccc}
\mathrm{e}^{i \boldsymbol{q}_{z 1}^{(n)} d^{(n)}} & 0 & 0 & 0 \\
0 & \mathrm{e}^{i \boldsymbol{q}_{z 2}^{(n)} d^{(n)}} & 0 & 0 \\
0 & 0 & \mathrm{e}^{i \boldsymbol{q}_{z 3}^{(n)} d^{(n)}} & 0 \\
0 & 0 & 0 & \mathrm{e}^{i \boldsymbol{q}_{z 4}^{(n)} d^{(n)}}
\end{array}\right],
\end{gathered}
$$

where $\boldsymbol{h}_{j}^{(n)}$ are the magnetic field eigen-polarizations and $d^{(n)}$ stands for the thickness of $n$-th layer. Each layer can be described by its own characteristic matrix $\mathbf{T}^{(n)}=$ $\mathbf{D}^{(n)} \mathbf{P}^{(n)}\left[\mathbf{D}^{(n)}\right]^{-1}$. All of the layers contained within the structure can be recursively connected and the amplitudes $\boldsymbol{A}^{(0)}, \boldsymbol{A}^{(N+1)}$ are related as $\boldsymbol{A}^{(0)}=\mathbf{M} \boldsymbol{A}^{(N+1)}$, where $\mathbf{M}$ is the total matrix:

$$
\mathbf{M}=\left[\mathbf{D}^{(0)}\right]^{-1}\left\{\prod_{l=1}^{N} \mathbf{T}^{(l)}\right\} \mathbf{D}^{(N+1)} .
$$

Alternatively, the scattering matrix $\mathbf{S}$ of the system can be derived in a similar way, relating out-going and incoming amplitudes: $\boldsymbol{A}_{\text {out }}=\mathbf{S} \boldsymbol{A}_{\text {in }}$.

\section{Appendix B: Calculation of field decay rate $\kappa$}

\section{Standard method based on complex frequency}

The total field decay rate $\kappa$ can be calculated rigorously within the framework of transfer or scattering matrix formalism. It can be done by using the formalism developed in Sec. III. However, the gain of active layer must be switched off $\bar{\chi}=0$ and the complex frequency $\tilde{\omega}=\omega-i \kappa$ must be introduced. In the context of given formalism it has more a sense of artificial gain according to sign convention. Similarly, also in this case, one is trying to find the values of $\omega$ and $\kappa$, which satisfy the

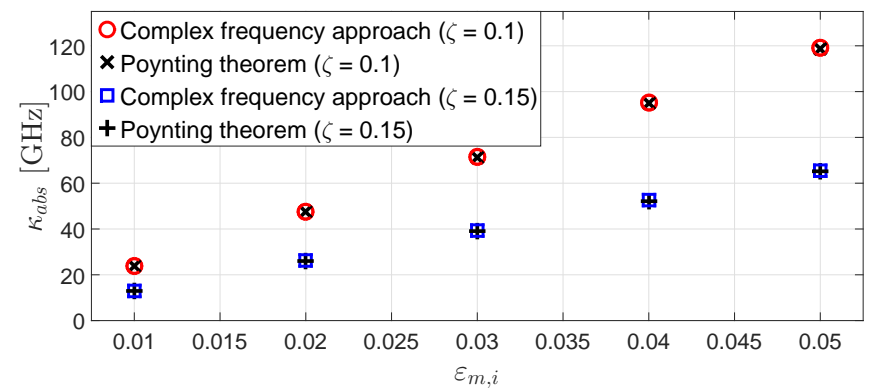

FIG. 11. Calculation of cavity decay rate contribution coming from internal absorptions $\kappa_{a b s}$ as a function of imaginary part of permittivity $\varepsilon_{m, i}$. Robust method based on matrix formalism is compared to results obtained using simple analytic expression.

resonance condition [see (27) and (29)]. When $\kappa$ reaches certain value, which is equal real cavity decay rate, the energy of considered cavity mode no longer decay [27, 69].

\section{Analytic method based on Poynting theorem}

Similarly, as in the case of anisotropy rates derived using couple mode theory, it is possible to derive simple analytic expressions also for $\kappa$. We combine the basic idea behind CMT, that one can assume the shape of laser mode, with the law of electromagnetic energy conservation, which is the Poynting theorem [70]:

$$
\frac{\partial}{\partial t} \int_{C} u \mathrm{~d} V=-\oint_{\partial C} \boldsymbol{S} \mathrm{d} \boldsymbol{A}-\int_{C} \boldsymbol{j} \boldsymbol{E} \mathrm{d} V
$$

where $u$ is the density of electromagnetic field, $\boldsymbol{S}$ is the Poynting vector and $\boldsymbol{j}$ stands for the density of electric current. If we consider the passive cavity, then it describes decay of the field due to transmitted light from the structure and internal absorptions. Let's consider the cavity mode linearly polarized along $\boldsymbol{x}$ or $\boldsymbol{y}$, described by $\boldsymbol{E}(z, t)=E(t) \boldsymbol{\varphi}(z)$. Thus, any lateral effects are neglected now. Using the known shape of cavity mode inside the device, the following relations can be derived. Density of electromagnetic field inside cavity with no magnetically active media is $u=\boldsymbol{E} \boldsymbol{D}=$ $\varepsilon_{0} \varepsilon_{m, r}|\varphi|^{2} E^{2}$, where $\varepsilon_{m, r}$ is the real part of spatiallyvarying permittivity. Stokes vector is then $\boldsymbol{S}=\boldsymbol{E} \times \boldsymbol{H}=$ $\left(\varepsilon_{0} c^{2} / \omega\right)|\varphi|^{2} E^{2} \boldsymbol{q}$. Absorbing term under the integral is equal $\boldsymbol{j} \boldsymbol{E}=\varepsilon_{0} \varepsilon_{m, i} \omega|\boldsymbol{\varphi}|^{2} E^{2}$, where $\varepsilon_{m, i}$ is the imaginary part of permittivity.

Assuming, that entire multilayer structure has the 
length $L$, one obtains:

$$
\begin{array}{r}
\int_{0}^{\mathcal{A}_{0}} \int_{0}^{L} \varepsilon_{m, r}|\boldsymbol{\varphi}|^{2} \mathrm{~d} z \mathrm{~d} \mathcal{A} \times \frac{\partial E}{\partial t}= \\
-\oint_{\partial C} \frac{c^{2}}{\omega}|\boldsymbol{\varphi}|^{2}(\boldsymbol{q} \mathrm{d} \mathcal{A}) \times E \\
-\int_{0}^{\mathcal{A}_{0}} \int_{0}^{L} \varepsilon_{m, i} \omega|\boldsymbol{\varphi}|^{2} \mathrm{~d} z \mathrm{~d} \mathcal{A} \times E,
\end{array}
$$

where $\mathcal{A}_{0}$ is the cross-sectional area of domain, over which the surface integration is performed. Simplifying this expression, one obtains a simple decay law

$$
\frac{\partial}{\partial t} E=-\underbrace{\left(\kappa_{t r}+\kappa_{a b s}\right)}_{\kappa} E,
$$

in which the particular contributions to total decay rate $\kappa$ consists of transmission term:

$$
\kappa_{t r}=\frac{c}{2} \frac{\sqrt{\varepsilon_{m, r}(0)}|\boldsymbol{\varphi}(0)|^{2}+\sqrt{\varepsilon_{m, r}(L)}|\boldsymbol{\varphi}(L)|^{2}}{\int_{C} \varepsilon_{m, r}(z)|\boldsymbol{\varphi}(z)|^{2} \mathrm{~d} z}
$$

and absorption term:

$$
\kappa_{a b s}=\frac{\omega}{2} \frac{\int_{a b s} \varepsilon_{m, i}(z)|\boldsymbol{\varphi}(z)|^{2} \mathrm{~d} z}{\int_{C} \varepsilon_{m, r}(z)|\boldsymbol{\varphi}(z)|^{2} \mathrm{~d} z} .
$$

By $\varepsilon_{m, r}(0)$ and $\varepsilon_{m, r}(L)$ we understand real parts of permittivity of the superstrate and substrate, respectively.
In the following, we focus on the $\kappa_{a b s}$ contributions, because it can be significantly simplified in the case of single absorbing layer. Spatial dependence of $\varepsilon_{m, i}$ can be taken out of the integral and we obtain:

$$
\kappa_{a b s}=\Gamma_{\kappa}\left(\frac{\omega}{2}\right) \varepsilon_{m, i}
$$

where the following confinement factor has been defined $\Gamma_{\kappa}=\int_{a b s}|\varphi(z)|^{2} \mathrm{~d} z / \int_{C} \varepsilon_{m, r}(z)|\varphi(z)|^{2} \mathrm{~d} z$. Derived expression is formally almost identical to approximate formulas, which have been derived for anisotropy rates $\gamma_{a, p}$.

We compare derived expression with the rigorous and more powerful method based on transfer (or scattering) matrix formalism. The results, which are in perfect agreement, are shown in Fig. 11.

\section{ACKNOWLEDGMENTS}

We thank Fabien Bretenaker for fruitful discussions. The support from the projects IT4 Innovations National Supercomputing Center Path to Exascale Project No. CZ.02.1.01/0.0/0.0/16 013/0001791, Student Project No. SP2020/150 and Czech Science Foundation Grant No. 18-22102S is acknowledged.
[1] F. Meier and B. Zakharchenya, Optical Orientation (North Holland, 1984).

[2] M. Holub and P. Bhattacharya, J. Phys. D: Appl. Phys. 40, R179 (2007).

[3] N. C. Gerhardt and M. R. Hofmann, Adv. Opt. Technol. 2012, 10.1155/2012/268949 (2012).

[4] M. Holub, J. Shin, S. Chakrabarti, and P. Bhattacharya, Appl. Phys. Lett. 87, 091108 (2005).

[5] S. Iba, S. Koh, K. Ikeda, and H. Kawaguchi, Appl. Phys. Lett. 98, 081113 (2011).

[6] J. Rudolph, S. Dhrmann, D. Hgele, M. Oestreich, and W. Stolz, Appl. Phys. Lett. 87, 241117 (2005).

[7] M. Holub and B. T. Jonker, Phys. Rev. B 83, 125309 (2011).

[8] J. Frougier, G. Baili, M. Alouini, I. Sagnes, H. Jaffrès, A. Garnache, C. Deranlot, D. Dolfi, and J.-M. George, Appl. Phys. Lett. 103, 252402 (2013).

[9] B. R. Cemlyn, I. D. Henning, M. J. Adams, E. Harbord, R. Oulton, V. Korpijrvi, and M. Guina, IEEE J. Quantum Electron. 55, 1 (2019).

[10] B. Cemlyn, M. Adams, E. Harbord, N. Li, I. Henning, R. Oulton, V.-M. Korpijrvi, and M. Guina, Semicond. Sci. Technol. 33, 094005 (2018).

[11] N. C. Gerhardt, M. Y. Li, H. Jähme, H. Höpfner, T. Ackemann, and M. R. Hofmann, Appl. Phys. Lett. 99, 151107 (2011).

[12] N. Yokota, K. Nisaka, H. Yasaka, and K. Ikeda, Appl.
Phys. Lett. 113, 171102 (2018)

[13] M. Lindemann, G. Xu, T. Pusch, R. Michalzik, M. Hofmann, I. Žutić, and N. Gerhardt, Nature 568, 1 (2019).

[14] R. Safian, G. Ghazi, and N. Mohammadian, Opt. Eng. 58, 1 (2019).

[15] M. Alouini, J. Frougier, A. Joly, G. Baili, D. Dolfi, and J.-M. George, Opt. Express 26, 6739 (2018).

[16] J. Frougier, G. Baili, I. Sagnes, D. Dolfi, J.-M. George, and M. Alouini, Opt. Express 23, 9573 (2015).

[17] A. Joly, G. Baili, M. Alouini, J.-M. George, I. Sagnes, G. Pillet, and D. Dolfi, Opt. Lett. 42, 651 (2017).

[18] T. Fördös, K. Postava, H. Jaffrès, and J. Pištora, J. Opt. 16, 065008 (2014).

[19] T. Fördös, H. Jaffrès, K. Postava, M. S. Seghilani, A. Garnache, J. Pištora, and H.-J. Drouhin, Phys. Rev. A 96, 043828 (2017).

[20] H. Benisty, R. Stanley, and M. Mayer, J. Opt. Soc. Am. A 15, 1192 (1998).

[21] R. Michalzik, VCSELs: Fundamentals, Technology and Applications of Vertical-Cavity Surface-Emitting Lasers (Springer-Verlag Berlin Heidelberg, 2013).

[22] T. Fördös, K. Postava, H. Jaffrès, D. Quang To, J. Pištora, and H. J. Drouhin, Appl. Phys. Lett. 112, 221106 (2018).

[23] S. Balle, Phys. Rev. A 57, 1304 (1998).

[24] G. Van der Sande, J. Danckaert, I. Veretennicoff, K. Panajotov, and S. Balle, Phys. Rev. A 71, 063801 
(2005).

[25] P. E. Faria Junior, G. Xu, J. Lee, N. C. Gerhardt, G. M. Sipahi, and I. Žutić, Phys. Rev. B 92, 075311 (2015).

[26] A. Y. Song, A. R. K. Kalapala, W. Zhou, and S. Fan, Appl. Phys. Lett. 113, 041106 (2018).

[27] H. E. Türeci, A. D. Stone, and B. Collier, Phys. Rev. A 74, 043822 (2006).

[28] L. Ge, Y. D. Chong, and A. D. Stone, Phys. Rev. A 82, 063824 (2010).

[29] S. Esterhazy, D. Liu, M. Liertzer, A. Cerjan, L. Ge, K. G. Makris, A. D. Stone, J. M. Melenk, S. G. Johnson, and S. Rotter, Phys. Rev. A 90, 023816 (2014).

[30] M. San Miguel, Q. Feng, and J. V. Moloney, Phys. Rev. A 52, 1728 (1995).

[31] J. Martin-Regalado, F. Prati, M. S. Miguel, and N. B. Abraham, IEEE J. Quantum Electron. 33, 765 (1997).

[32] M. Travagnin, M. P. van Exter, A. K. Jansen van Doorn, and J. P. Woerdman, Phys. Rev. A 54, 1647 (1996).

[33] M. Travagnin, Phys. Rev. A 56, 4094 (1997).

[34] M. Adams, N. Li, B. Cemlyn, H. Susanto, and I. Henning, Semicond. Sci. Technol. 33, 064002 (2018).

[35] A. K. Jansen van Doorn, M. P. van Exter, and J. P. Woerdman, Appl. Phys. Lett. 69, 3635 (1996).

[36] S. Balle, E. Tolkachova, M. S. Miguel, J. R. Tredicce, J. Martín-Regalado, and A. Gahl, Opt. Lett. 24, 1121 (1999).

[37] D. Burak, J. V. Moloney, and R. Binder, Phys. Rev. A 61, 053809 (2000).

[38] J. Mulet and S. Balle, IEEE J. Quantum Electron. 38, 291 (2002).

[39] M. S. Torre, C. Masoller, and P. Mandel, Phys. Rev. A 74, 043808 (2006).

[40] A. Valle, M. Sciamanna, and K. Panajotov, Phys. Rev. E 76, 046206 (2007).

[41] K. Böhringer and O. Hess, Prog. Quantum. Electron. 32, 159 (2008).

[42] K. Böhringer and O. Hess, Prog. Quantum. Electron. 32, 247 (2008)

[43] M. Vaughan, H. Susanto, I. Henning, and M. Adams, IEEE J. Quantum Electron. , 11 (2020).

[44] S. E. Hodges, M. Munroe, J. Cooper, and M. G. Raymer, J. Opt. Soc. Am. B 14, 191 (1997).

[45] H. Haken, Light II: Laser Light Dynamics (NorthHolland Publishing Company, 1985).

[46] M. Sargent, M. O. Scully, and W. E. Lamb, Laser physics (London : Addison-Wesley, 1974).

[47] S. V. Zhukovsky, D. N. Chigrin, and J. Kroha, Phys. Rev. A 79, 033803 (2009).

[48] R. Paquet, S. Blin, M. Myara, L. L. Gratiet, M. Sellahi, B. Chomet, G. Beaudoin, I. Sagnes, and A. Garnache, Opt. Lett. 41, 3751 (2016).

[49] S. Blin, R. Paquet, M. Myara, B. Chomet, L. Le Gratiet, M. Sellahi, G. Beaudoin, I. Sagnes, G. Ducournau, P. Latzel, J. Lampin, and A. Garnache, IEEE J. Sel. Topics Quantum Electron. 23, 1 (2017).
[50] M. Drong, T. Fördös, H. Y. Jaffrès, J. Peřina, K. Postava, J. Pištora, and H.-J. Drouhin, J. Opt. 22, 055001 (2020).

[51] J.-L. Yu, Y.-H. Chen, C.-G. Tang, C. Jiang, and X.-L. Ye, Nanoscale Res. Lett. 6, 210 (2011).

[52] O. Krebs and P. Voisin, Phys. Rev. Lett. 77, 1829 (1996).

[53] M. S. Park, B. T. Ahn, B.-S. Yoo, H. Y. Chu, H.-H. Park, and C. J. Chang-Hasnain, Appl. Phys. Lett. 76, 813 (2000).

[54] A. Siegman, Lasers (University Science Books, 1986).

[55] P. Yeh, Optical Waves in Layered Media (John Wiley and Sons, 1988).

[56] C. Henry, IEEE J. Quantum Electron. 18, 259 (1982).

[57] M. Sargent, W. E. Lamb, and R. L. Fork, Phys. Rev. 164, 436 (1967).

[58] M. S. Seghilani, Highly coherent III-V-semiconductor laser emitting phase-, amplitude- and polarizationstructured light for advanced sensing applications: Vortex, SPIN, Feedback Dynamics, Ph.D. thesis, Université de Montpellier (2015).

[59] S. Adachi, J. Appl. Phys. 66, 6030 (1989).

[60] T. Henn, L. Czornomaz, and G. Salis, Appl. Phys. Lett. 109, 152104 (2016).

[61] T. Pusch, S. Scherbl, M. Lindemann, N. C. Gerhardt, M. R. Hofmann, and R. Michalzik, in Semiconductor Lasers and Laser Dynamics VIII, Vol. 10682, edited by K. Panajotov, M. Sciamanna, and R. Michalzik, International Society for Optics and Photonics (SPIE, 2018) pp. $120-125$.

[62] T. Pusch, P. Debernardi, M. Lindemann, F. Erb, N. C. Gerhardt, M. R. Hofmann, and R. Michalzik, Electron. Lett. 55, 1055 (2019).

[63] T. Fördös, E. Clarke, P. Patil, R. J. Airey, N. Babazadeh, B. Cemlyn, M. Adams, I. Henning, and J. Heffernan, in 2019 24th Microoptics Conference (MOC) (2019) pp. 306-307.

[64] K. Hirose, Y. Liang, Y. Kurosaka, A. Watanabe, T. Sugiyama, and S. Noda, Nat. Photonics 8, 406 (2014).

[65] K. J. Reilly, A. Kalapala, S. Yeom, S. J. Addamane, E. Renteria, W. Zhou, and G. Balakrishnan, J. Cryst. Growth 535, 125531 (2020).

[66] S. Tang, B. Zhu, M. Jia, Q. He, S. Sun, Y. Mei, and L. Zhou, Phys. Rev. B 91, 174201 (2015).

[67] M. Foldyna, R. Ossikovski, A. D. Martino, B. Drevillon, K. Postava, D. Ciprian, J. Pištora, and K. Watanabe, Opt. Express 14, 3114 (2006).

[68] K. Postava, T. Fördös, H. Jaffrès, L. Halagačka, H. J. Drouhin, and J. Pištora, Proc. SPIE, Integrated Optics: Physics and Simulations II 9516, 153 (2015).

[69] T. Harayama, S. Sunada, and K. S. Ikeda, Phys. Rev. A 72, 013803 (2005).

[70] R. P. Feynman, R. B. Leighton, and M. Sands, The Feynman Lectures on Physics, Volume II (Addison-Wesley, 1966). 